\title{
Talin2-mediated traction force drives matrix degradation and cell invasion
}

\author{
Lei Qi ${ }^{1,2, *}$, Naser Jafari ${ }^{1,2, *}$, Xiang Li ${ }^{1, *}$, Zaozao Chen ${ }^{3}$, Liqing $\mathrm{Li}^{1}$, Vesa P. Hytönen ${ }^{4}$, Benjamin T. Goult ${ }^{5}$, \\ Chang-Guo Zhan ${ }^{6}$ and Cai Huang ${ }^{1,2,7, \pm}$
}

\begin{abstract}
Talin binds to $\beta$-integrin tails to activate integrins, regulating cell migration, invasion and metastasis. There are two talin genes, TLN1 and TLN2, encoding talin1 and talin2, respectively. Talin1 regulates focal adhesion dynamics, cell migration and invasion, whereas the biological function of talin2 is not clear and, indeed, talin2 has been presumed to function redundantly with talin1. Here, we show that talin2 has a much stronger binding to $\beta$-integrin tails than talin1. Replacement of talin2 Ser339 with Cys significantly decreased its binding to $\beta 1$-integrin tails to a level comparable to that of talin1. Talin2 localizes at invadopodia and is indispensable for the generation of traction force and invadopodium-mediated matrix degradation. Ablation of talin2 suppressed traction force generation and invadopodia formation, which were restored by re-expressing talin2 but not talin1. Furthermore, re-expression of wild-type talin2 (but not talin2 ${ }^{\mathrm{S} 339 \mathrm{C}}$ ) in talin2-depleted cells rescued development of traction force and invadopodia. These results suggest that a strong interaction of talin2 with integrins is required to generate traction, which in turn drives invadopodium-mediated matrix degradation, which is key to cancer cell invasion.
\end{abstract}

KEY WORDS: Talin1, Talin2, Traction force, Focal adhesions, Cell invasion

\section{INTRODUCTION}

Talin activates integrin and plays a pivotal role in cell migration, invasion and cancer metastasis (Desiniotis and Kyprianou, 2011; Huang et al., 2009; Jin et al., 2015; Tadokoro et al., 2003). Talin contains an N-terminal globular head domain and a C-terminal rod domain (Goult et al., 2013; Nuckolls et al., 1990). The talin head domain contains a FERM domain and is responsible for the binding of talin to $\beta$-integrin tails (Calderwood et al., 1999). The rod domain has several vinculin-binding sites and two actin-binding sites (Atherton et al., 2015; Gingras et al., 2005; Hemmings et al., 1996). The binding of talin to $\beta$-integrin tails is essential for integrin activation (Calderwood et al., 1999; Tadokoro et al., 2003), which

\footnotetext{
${ }^{1}$ Markey Cancer Center, University of Kentucky, Lexington, KY 40506, USA

${ }^{2}$ Veterans Affairs Medical Center, Lexington, KY 40502, USA. ${ }^{3}$ Department of Cell Biology \& Physiology, University of North Carolina at Chapel Hill, Chapel Hill, NC 27599, USA. ${ }^{4}$ BioMediTech, University of Tampere, 33520 Tampere, Finland and Fimlab Laboratories, Tampere 33520, Finland. ${ }^{5}$ School of Biosciences, University of Kent, Canterbury, Kent CT2 7NJ, UK. ${ }^{6}$ Molecular Modeling and Biopharmaceutical Center, College of Pharmacy, University of Kentucky, Lexington, KY 40506, USA. Department of Pharmacology and Nutritional Sciences, University of Kentucky, Lexington, KY 40506, USA.

*These authors contributed equally to this work

${ }^{\ddagger}$ Author for correspondence (cai-huang@uky.edu)

(iD) C.H., 0000-0003-4258-3841
}

Received 12 January 2016; Accepted 18 August 2016 in turn regulates focal adhesion dynamics and invadopodium formation (Bate et al., 2012; Beaty et al., 2014; Franco et al., 2004; Nayal et al., 2004) - key steps in cell migration and invasion (Beaty and Condeelis, 2014; Saykali and El-Sibai, 2014; Webb et al., 2002; Wehrle-Haller, 2012). Talin also mediates calpain-induced focal adhesion disassembly (Bate et al., 2012; Franco et al., 2004). Talin1 phosphorylation by $\mathrm{Cdk} 5$ regulates focal adhesion dynamics, integrin activation, cell migration, invasion and metastasis (Huang et al., 2009; Jin et al., 2015). Talin interacts with phosphatidylinositol phosphate kinase $\gamma$, which produces phosphatidylinositol 4,5-bisphosphate (PIP2) to regulate focal adhesion dynamics, cell migration and invasion (Chen et al., 2015; Di Paolo et al., 2002; Li et al., 2013; Ling et al., 2002; Wu et al., 2011). It recruits the moesin-NHE1 complex to modulate $\mathrm{pH}$ at invadopodia, consequently governing invadopodium stability and matrix degradation (Beaty et al., 2014). It is also required for the initial generation of mechanical force (Giannone et al., 2003; Zhang et al., 2008).

There are two talin genes, TLN1 and TLN2, encoding talin 1 and talin2, respectively. Talin1 has been well studied, whereas the biological function of talin2 is less clear. It has been shown that talin2 regulates focal adhesion assembly and focal adhesion kinase (FAK) signaling in the absence of talin1 (Zhang et al., 2008). Talin2 is usually localized at large focal adhesions and fibrillar adhesions, whereas talin 1 is usually found at smaller focal adhesions in the peripheral region (Praekelt et al., 2012; Senetar et al., 2007). Trastuzumab, an antibody therapeutic targeting HER2 for the treatment of cancer, inhibits cell migration and invasion by downregulating talin2 (Le et al., 2012). Nevertheless, it has been reported that depletion of talin 2 does not influence $\beta 1$-integrin activation (Jin et al., 2015). Thus, it had been presumed that talin2 functions redundantly with talin 1 .

In the present study, we demonstrate that talin2 functions distinctly from talin 1 as a regulator of cell invasion. We show that talin 2 has a stronger interaction with $\beta$-integrin tails than talin 1 , and that it colocalizes with invadopodia, regulates the generation of traction force and invadopodium-mediated matrix degradation. Furthermore, the strong interaction of talin 2 with $\beta$-integrin tails is essential for the development of strong traction and invadopodia, as replacement of Ser339 with Cys in the talin2 head domain compromises binding to $\beta$-integrin tails, and inhibits traction force and invadopodium-mediated matrix degradation. Recently, a disease-causing missense variant, S339L, of the TLN2 gene has been identified as the cause of fifth finger camptodactyly, a digit deformity in humans (Deng et al., 2016), which occurred in the presence of talin1, further highlighting distinct roles of talin1 and talin2 in development. This also demonstrates the physiological relevance of residue S339 in talin2 function and the requirement for strong talin2-integrin linkage in normal development. Thus, interaction of talin 2 with integrins is required for the generation of 
traction force, which in turn drives invadopodium-mediated matrix degradation, and ultimately, cancer cell invasion.

\section{RESULTS}

\section{Talin2 binds to $\boldsymbol{\beta}$-integrin tails more strongly than talin1}

To learn whether talin 1 and talin 2 have any difference in binding to $\beta$-integrin tails, CHO-K1 cells were transfected with EGFP-talinhead constructs - EGFP-talin $1_{1-433}$, EGFP-talin $1_{1-446}$ or EGFPtalin $2_{1-449}$ - and binding to integrin tails was determined by GST- $\beta$-integrin-tail pulldown assays, using GST as a control. The interaction of talin $2_{1-449}$ with the tail of $\beta 1 \mathrm{~A}$-integrin (an isoform encoded by $I T G B 1$ ) was more efficient than that of either talin $1_{1-433}$ or talin $1_{1-446}$ (Fig. 1A). Similar results were observed with the binding of the tails of the $\beta 3 \mathrm{~A}$-integrin and $\beta 1 \mathrm{D}$-integrin (isoforms encoded by $I T G B 3$ and $I T G B 1$, respectively) (Fig. S1A). Deletion of the $\mathrm{C}$-terminus of the FERM domain enhanced the binding of talin $2_{1-449}$ to $\beta 1 \mathrm{~A}$-integrin tails (Fig. 1B) but reduced the binding of talin $1_{1-446}$ (Fig. 1C). The difference between talin 1 and talin2 in integrin binding was not caused by other associated proteins in the cell lysates, because purified His-tagged talin $2_{1-449}$ binding to $\beta 1 \mathrm{~A}-$ integrin tail was also stronger than that of purified talin $1_{1-443}$ and talin $1_{1-446}$ (Fig. 1D). Thus, the talin2 head domain (TH2) has a much higher affinity than the talin1 head domain (TH1) for $\beta$-integrin tails.

\section{Talin1 residue $\mathrm{C} 336$ and talin2 residue $S 339$ contribute to the isoform-specific affinity differences}

To identify why talin 2 binding to integrins is stronger than talin1, we aligned the sequences of talin 1 and talin 2 around the integrinbinding site in the F3 FERM subdomain. There are several residues that are different between the integrin-binding sequences of talin1 and talin2 (Fig. 1E). Replacement of Cys336 of talin1 with Ser enhanced the binding of talin 1 to the $\beta 1 \mathrm{~A}$-integrin tail to an extent comparable to that of talin2 (Fig. 1F), whereas replacement of Ser339 on talin2 with Cys substantially attenuated its binding (Fig. 1G). Replacement of Cys336 on talin1 with other residues dramatically changed its binding to the $\beta 1 \mathrm{~A}$-integrin tail (Fig. S1B), whereas mutation at several other mismatching residues had no effect on the ability of talin1 to bind to integrin tails (data not shown). The recent finding that mutation of Ser339 to Leu in talin2 has a disease-causing phenotype in humans, giving rise to the pathology fifth finger camptodactyly, highlights the physiological importance of this residue. Indeed, a S339L mutant has a similar effect to that of S339C on integrin binding (Fig. 1H). Similar results were observed in pulldown assays using purified His-tagged $T \ln 1_{1-446}, T \ln 2_{1-449}$ and mutants (Fig. 1I). These data indicate that Ser339 plays an important role in the high integrin-binding ability of talin2.

\section{Talin2 is essential for the assembly of large, stable focal adhesions and generation of traction force}

To ascertain whether talin 2 is different from talin 1 in regulating focal adhesions, we used CRISPR-Cas9 to knockout talin1 or talin2 from U2OS cells. Ablation of talin isoforms was achieved by infecting the cells with lentiviruses that express Cas9 and talin1 or talin2 guide (g)RNAs (Fig. 2A). The talin1- or talin2-null (knockout) cells were plated on fibronectin and stained for zyxin and either talin1 or talin2, using cells expressing empty LentiCRISPR v2 vector as a control. Ablation of either talin1 or talin2 inhibited the assembly of small focal adhesions $\left(<7 \mu \mathrm{m}^{2}\right)$, as determined with staining of zyxin staining (Fig. 2B and $\mathrm{C}$ ). However, ablation of talin 1 did not influence large focal adhesions $\left(>10 \mu \mathrm{m}^{2}\right)$, whereas knockout of talin2 significantly diminished the formation of large focal adhesions (Fig. 2C, bottom panel). Similar results were observed in cells where talin 1 or talin 2 were depleted using small hairpin (sh)RNAs (Fig. S2A-C). In the central regions ( $>5 \mu \mathrm{m}$ from edges) of U2OS cells, a higher number of large spots of talin2 were visible than those of talin1 (Fig. 2D). Likewise, in MDA-MB-231 and MDA-MB-435S cancer cells, talin1 was usually found in smaller focal adhesions, whereas talin2 formed larger focal adhesions (Fig. S2D and E). However, although ablation of talin 1 also confined the assembly of FAK to small focal adhesions $\left(<7 \mu \mathrm{m}^{2}\right)$, ablation of talin2 had little effect on FAK localization (Fig. 2E and F). These results suggest that talin2 is mainly responsible for large, stable focal adhesion assembly, whereas talin 1 mediates smaller focal adhesion formation.

To compare the roles of talin 1 and talin 2 in the generation of traction force, talin1- or talin2-ablated U2OS cells were plated onto fibronectin-conjugated polyacrylamide gels containing Red FluoSpheres, using cells carrying empty CRISPR vector as a control. Traction force was measured using a Nikon A1 confocal microscope equipped with a $\mathrm{CO}_{2}$ incubator system and analyzed using the method of Butler et al. (2002). Knockout of talin 1 or talin2 significantly inhibited the traction force generated (Fig. 3A and C). Talin1 knockout also significantly depressed the cell spreading on the polyacrylamide gel, whereas talin 2 knockout had only marginal effects (Fig. 3B), suggesting that the effect of talin1 knockout on traction force might be caused by suppression of cell spreading.

Because the TH1 has been reported to mediate cell spreading in talin1-knockout cells (Zhang et al., 2008), we examined whether transfection of TH1 could rescue the traction force production in talin1-null cells. Talin1-null U2OS cells were transfected with EGFP-talin $1_{1-446}$ (constituting TH1) or EGFP-talin2 ${ }_{1-449}$ (constituting TH2) (Fig. 4A). The cells were plated on polyacrylamide gels containing Red FluoSpheres for analysis in cell spreading and traction force assays, using talin1-knockout cells as a control. Transfection of talin1-knockout cells with TH1 significantly rescued the cell-spreading defect, whereas transfection with TH2 had no effect (Fig. 4B and C). TH1-mediated cell spreading was accompanied by an increase in traction force, whereas $\mathrm{TH} 2$ was unable to rescue the cell-spreading and traction-force defect caused by the ablation of talin1 (Fig. 4B and D). Because the talin head domain lacks the predominant actin-binding site (Atherton et al., 2015), it is deficient in mediating traction force production. These results suggest that the apparent role of talin 1 in the generation of traction force is partially caused by its effect on cell spreading.

To explore whether talin2 is dispensable for traction force generation, talin2-knockout U2OS cells were transfected with fulllength EGFP-talin1 or EGFP-talin2 (Fig. 4E), and the traction force in these cells was determined, using talin2-knockout cells and CRISPR vector cells as controls. Transfection with talin 1 caused only a slight increase in traction force, whereas transfection with talin2 almost restored the traction force to the levels of CRISPR control cells (Fig. 4F). The role of talin2 in traction force production is further supported by shRNA knockdown experiments, where depletion of talin 2 caused a significant reduction in traction force production in U2OS cells and MDA-MB-231 cells (Fig. S3). These results indicate that talin 2 is indispensable for cellular traction force generation.

\section{Strong binding of talin2 to integrins is essential for the development of traction force}

To stably express full-length talin2 and talin $2^{\mathrm{S} 339 \mathrm{C}}$ in talin2-null U2OS cells, pAAVS1-EGFP-talin $2^{\mathrm{WT}}$ or pAAVS1-EGFP- 

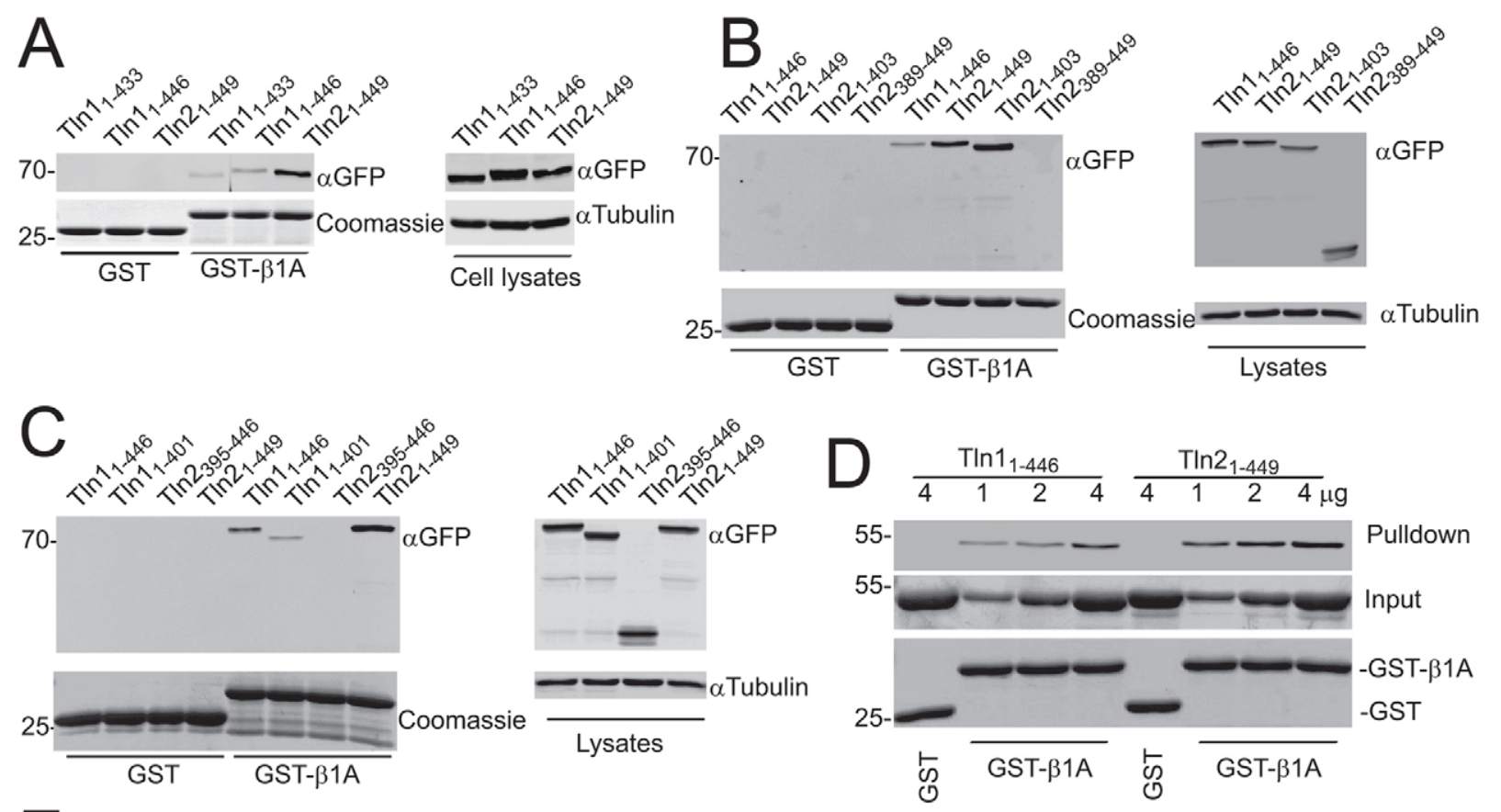

E

Ta1in1 321 GKNKLVPRLLGITKECVMRVDEKTKEVIQEWNLTNIKRWAASPKSFTLDFGDYQDGYYSV 380

|||||||||||||||||||||||||||||||||||||||||||||||||||||||||||||

Talin2 324 GKNKLVPRLLGITKDSVMRVDEKTKEVLQEWPLTTVKRWAASPKSFTLDFGEYQESYYSV 383
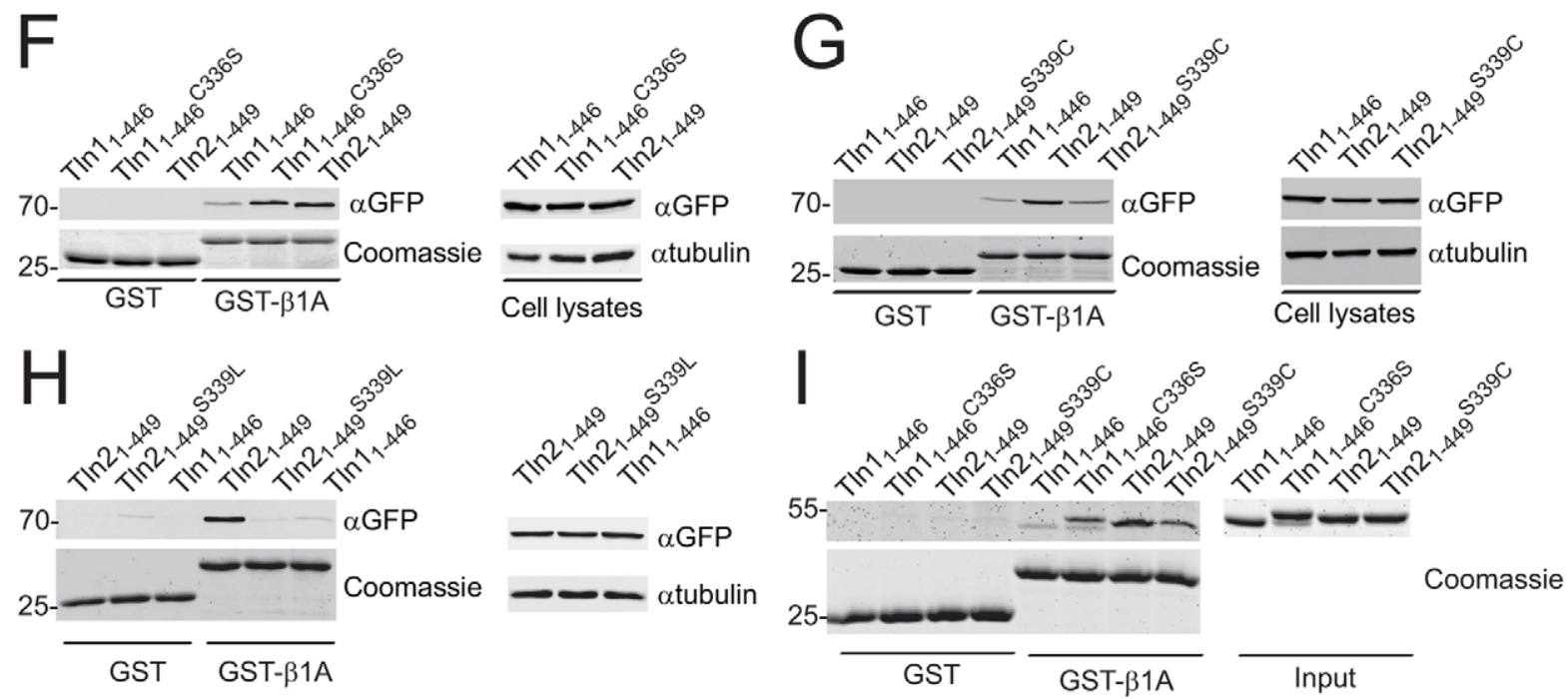

Fig. 1. Talin2 has a much higher affinity than talin1 for $\beta$-integrin tails. (A-C) Binding of talin-truncated mutants to $\beta 1 A$-integrin tails, measured by using GST pulldown assays. The EGFP fusion proteins of talin mutants were transiently expressed in CHO-K1 cells. (A) Binding of EGFP-talin ${ }_{1-433}\left(\right.$ TIn1 $\left._{1-433}\right)$,

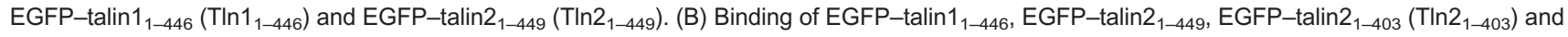

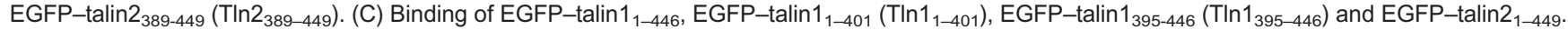
(D) Interaction of purified His-tagged talin $1_{1-446}$ and talin $2_{1-449}$ to immobilized GST and GST- $\beta 1$ A-integrin-tails. The binding was detected by Coomassie staining. (E) Sequence alignment of the integrin-binding region of the F3 FERM domains of talin1 and talin2. Residues C336 in talin1 and S339 in talin2 are shown in red. Yellow and red lines indicate unmatched residues with similar and different chemical properties, respectively. (F-H) The EGFP fusion proteins of talin mutants were transiently expressed in $\mathrm{CHO}-\mathrm{K} 1 \mathrm{cells}$. The binding of talin mutants to $\beta 1 \mathrm{~A}$-integrin tails was determined by GST pulldown assays. ( $\mathrm{F}$ ) Substitution of talin1 residue C336 with Ser promoted its binding to $\beta 1 \mathrm{~A}$-integrin tails. $(\mathrm{G})$ Replacement of talin2 S339 with Cys reduced its binding to $\beta 1 \mathrm{~A}$-integrin tails. (H) Replacement of talin2 S339 with Leu diminished its binding to $\beta 1$ A-integrin tails. (I) Binding of purified His-tagged talin $1_{1-446}$, talin $1_{1-446}{ }^{\mathrm{C} 336 \mathrm{~S}}$, talin2 ${ }_{1-449}$ and talin2 ${ }_{1-449}{ }^{\text {S339C }}$ to immobilized GST and GST- $\beta 1$ A-integrin-tails. Binding was detected by Coomassie staining.

talin2 ${ }^{\mathrm{S} 339 \mathrm{C}}$ were co-transfected with AAVS1 gRNA into talin2-null U2OS cells. The cells were selected with neomycin and sorted for EGFP-positive cells. The expression of EGFPtalin2 and EGFP-talin $2^{\mathrm{S} 339 \mathrm{C}}$ was examined by western blotting
(Fig. 5A). To examine whether the strong binding of talin2 to $\beta$-integrins is essential for traction force generation, talin2null cells that expressed EGFP-talin2 or EGFP-talin2 ${ }^{\mathrm{S} 339 \mathrm{C}}$ were plated onto the gelatin-conjugated polyacrylamide gels 

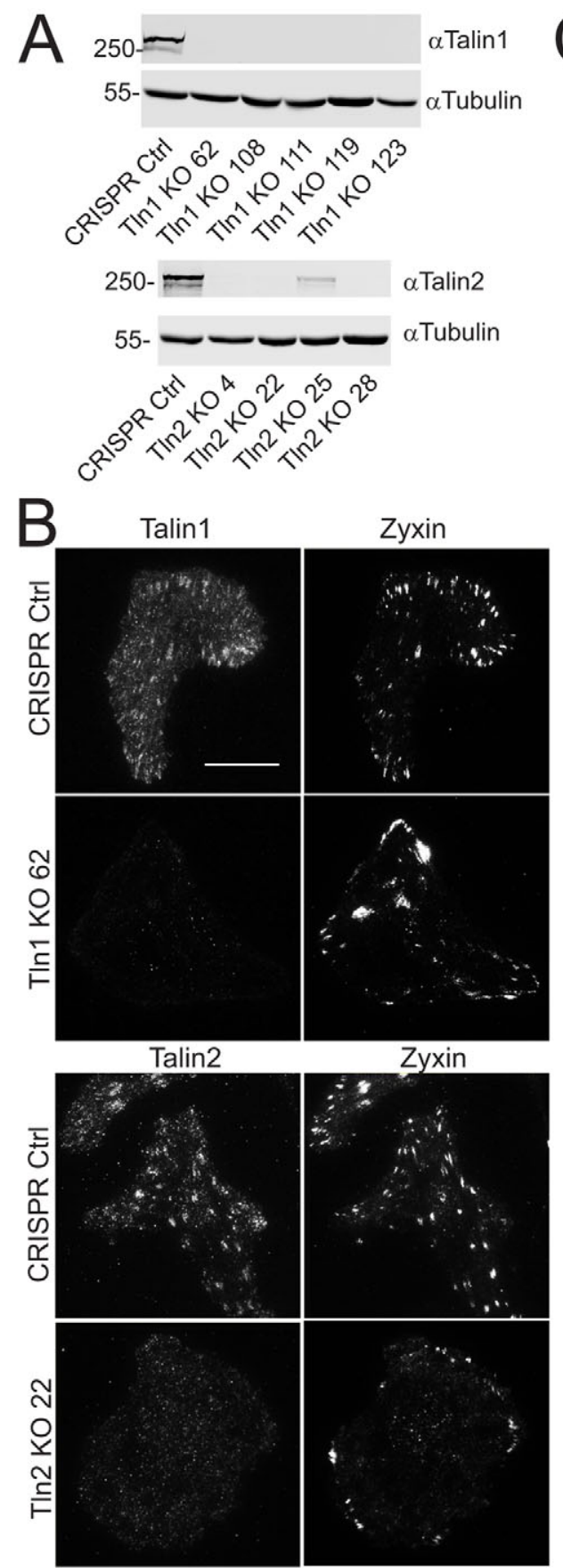

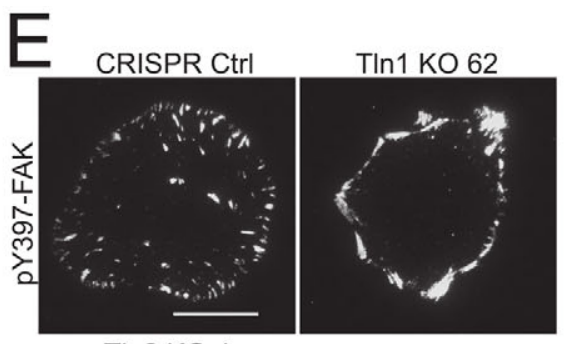

$\mathrm{TIn} 2 \mathrm{KO} 4$
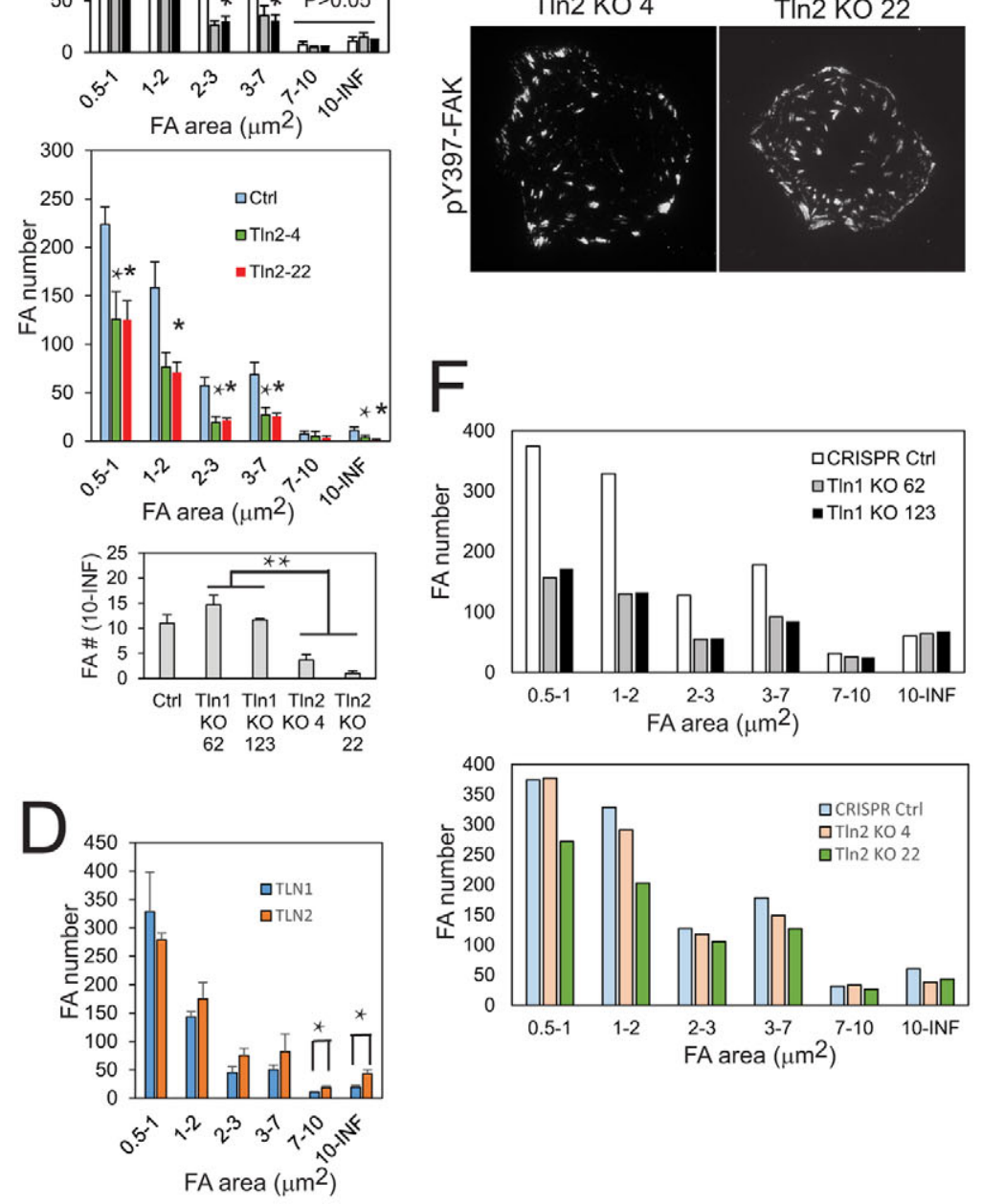

FA area $\left(\mu \mathrm{m}^{2}\right)$

Fig. 2. Talin1 is required for small focal adhesion formation, whereas talin2 is responsible for large, stable focal adhesion assembly. (A) Endogenous talin1 and talin2 in CRISPR-vector-transfected (CRISPR Ctrl) U2OS cells and talin1- or talin2-null (TIn1 KO and TIn2 KO, respectively) U2OS cells. Numbers represent different clones. (B) The distribution of zyxin and talin1 (top) or talin2 (bottom) in talin1-and talin2-null cells. Talin1- or talin2-null U2OS cells were plated onto fibronectin- $(5 \mu \mathrm{g} / \mathrm{ml})$ coated glass-bottomed dishes for $4 \mathrm{~h}$, fixed and co-stained for talin1 (or talin2) and zyxin. Images were acquired by using TIRF microscopy. Scale bar: $20 \mu \mathrm{m}$. (C) Area distribution of zyxin staining in talin1- (top) and talin2- (middle) null U2OS cells. The small graph at the bottom highlights the different effects of talin1 and talin2 knockout on large $\left(>10 \mu \mathrm{m}^{2}\right)$ focal adhesions. Data are mean \pm s.e.m. of three experiments. In each experiment, focal adhesions of 20 cells from each group were analyzed and plotted. 10-INF represents FAs larger than $10 \mu m^{2}$. TIn1-62, TIn1-123, TIn2-4 and TIn2-22

represent different clones of talin 1 or talin2 KO cells. $t$-test, ${ }^{*} P<0.05,{ }^{* *} P<0.01,{ }^{* * *} P<0.001$ against CRISPR control cells, unless specified otherwise in the figures. (D) Area distribution of talin1 and talin2 staining in central parts ( $5 \mu \mathrm{m}$ from edges) of cells. Data are mean \pm s.e.m. of three experiments. $t$-test, ${ }^{*} P<0.05$. (E) The distribution of phosphorylated FAK at Y397 (pY397-FAK) in talin1- or talin2-null cells. Talin1- or talin2-null U2OS cells were cultured on fibronectin, fixed and stained for pY397-FAK. Scale bar: $20 \mu \mathrm{m}$. (F) Area distribution of pY397-FAK staining in talin1- (left) and talin2- (right) null U2OS cells. Data are means of two experiments. In each experiment, focal adhesions of 20 cells from each group were analyzed and plotted.

containing Red FluoSpheres, and traction force was measured using talin2-null cells and CRISPR vector cells as controls. Expression of EGFP-talin2 in talin2-null cells restored more than $70 \%$ of the traction force as compared to that of
CRISPR vector cells, whereas that of EGFP-talin2 ${ }^{\mathrm{S} 339 \mathrm{C}}$, which has reduced affinity to $\beta$-integrins, had little effect (Fig. 5B and C). This result suggests that a strong binding of talin 2 to $\beta$-integrins is required for traction force generation. 

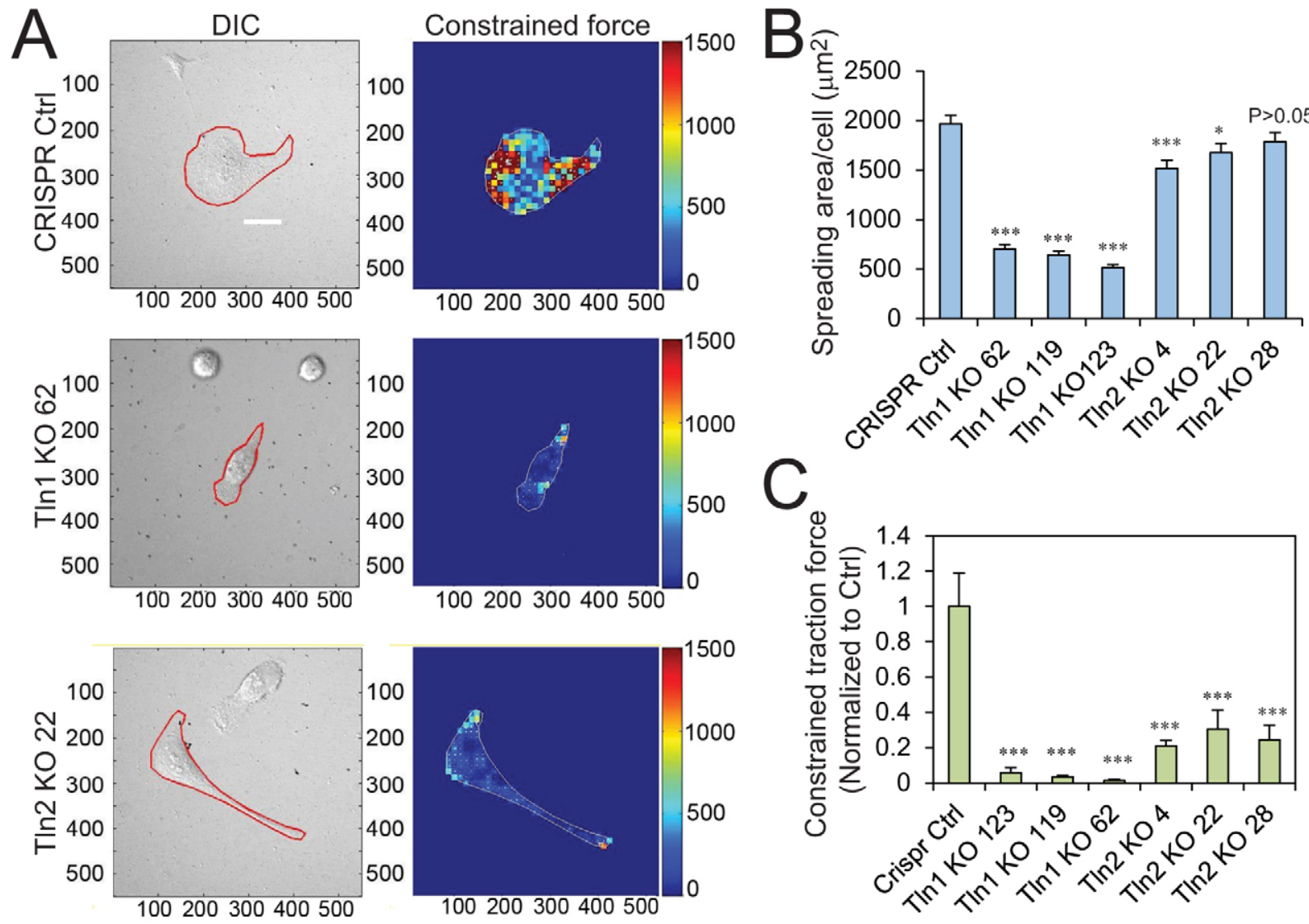

Fig. 3. The roles of talin1 and talin2 in cell spreading and traction force production. (A) Effects of talin1 and talin2 knockout (TIn1 KO 62 and TIn2 KO 22 , respectively) on cell spreading and traction force generation in U2OS cells. Axis labels are pixel numbers. Scale bar: $30 \mu m$. (B) Quantitative cell spreading area in talin1- or talin2-ablated U2OS cells, using cells carrying CRISPR vector (CRISPR Ctrl) as a control. Data are presented as mean \pm s.e.m. of three

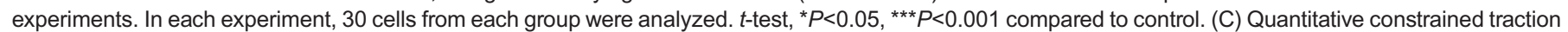
force in talin1- or talin2-ablated U2OS cells, using cells carrying CRISPR vector as a control. Data are presented as mean \pm s.e.m. of three independent experiments. $t$-test, ${ }^{* \star *} P<0.001$.

\section{Strong binding of talin2 to integrins is required for cell invasion}

To examine the role of talin1 and talin2 in cell invasion, talin1- or talin2-ablated U2OS cells were examined for their capacity to penetrate through Transwell filters coated with Matrigel. As shown in Fig. 6A and B, ablation of either talin1 or talin2 inhibited the invasion of U2OS cells. Knockout of talin1 or talin2 caused $\sim 77-91 \%$ inhibition of U2OS cell invasion, as compared to that of CRISPR vector cells. This crucial role of talin 1 and talin 2 in cell invasion was also observed in MDA-MB-231 cells (Fig. 6C). These results indicate that both talin1 and talin2 play crucial roles in cell invasion.

To determine the essential role of the talin $2-\beta$-integrin interaction in cell invasion, talin2-null U2OS cells that expressed EGFP-talin2 or EGFP-talin2 ${ }^{\mathrm{S} 339 \mathrm{C}}$ were tested for their invasive capacities toward Matrigel, using talin2-null cells and CRISPR vector cells as controls. Expression of EGFP-talin2 in talin2-null cells significantly rescued the capacity of the cells to invade, whereas expression of EGFPtalin $2^{\text {S339C }}$ had only a small effect (Fig. 6D and E), suggesting that strong binding of talin 2 to integrins is required for cell invasion.

\section{Talin2 colocalizes with invadopodia and regulates matrix degradation}

To examine the colocalization of talin2 with invadopodia, U2OS cells were plated on Cy3-labeled gelatin that was immobilized on glass- bottomed dishes, and cells were co-stained for talin2 and the invadopodium markers cortactin or Tks5. Talin2 significantly colocalized with cortactin and Tks5 at either large or small degradation holes (Fig. 7A and B). Only about $80 \%$ of invadopodia contained both cortactin and talin2, suggesting that talin 2 and cortactin contribute to different stages of invadopodia. MDA-MB-231 cells had more invadopodia, in which talin2 colocalized with cortactin, as compared to that observed in U2OS cells (Fig. 7C). Talin1 also colocalized with cortactin at invadopodia in MDA-MB-231 cells (Fig. S4A). We then examined the colocalization of talin2 with two other invadopodium markers - $\beta 1$-integrin and N-WASP. $\beta 1$-integrin and talin2 colocalized with gelatin degradation holes, whereas $\beta 1$-integrin at focal adhesions did not (Fig. S4B). Talin2 also colocalized with N-WASP at invadopodia-like structures (Fig. S4C). These results suggest that talin 2 is a marker for invadopodia.

To determine the roles of talin1 and talin2 in invadopodia, talin1or talin2-ablated U2OS cells were plated on Alexa-Fluor-488labeled gelatin and stained for filamentous actin. Although ablation of either talin 1 or talin 2 dramatically inhibited invadopodiummediated matrix degradation, knockout of talin 2 had a slightly more severe effect on invadopodia formation than did talin1 knockout (Fig. 7D and E), suggesting a key role of talin2 in invadopodium regulation.

To examine whether talin 2 is indispensable for invadopodiummediated matrix degradation, talin2-knockout U2OS cells were 

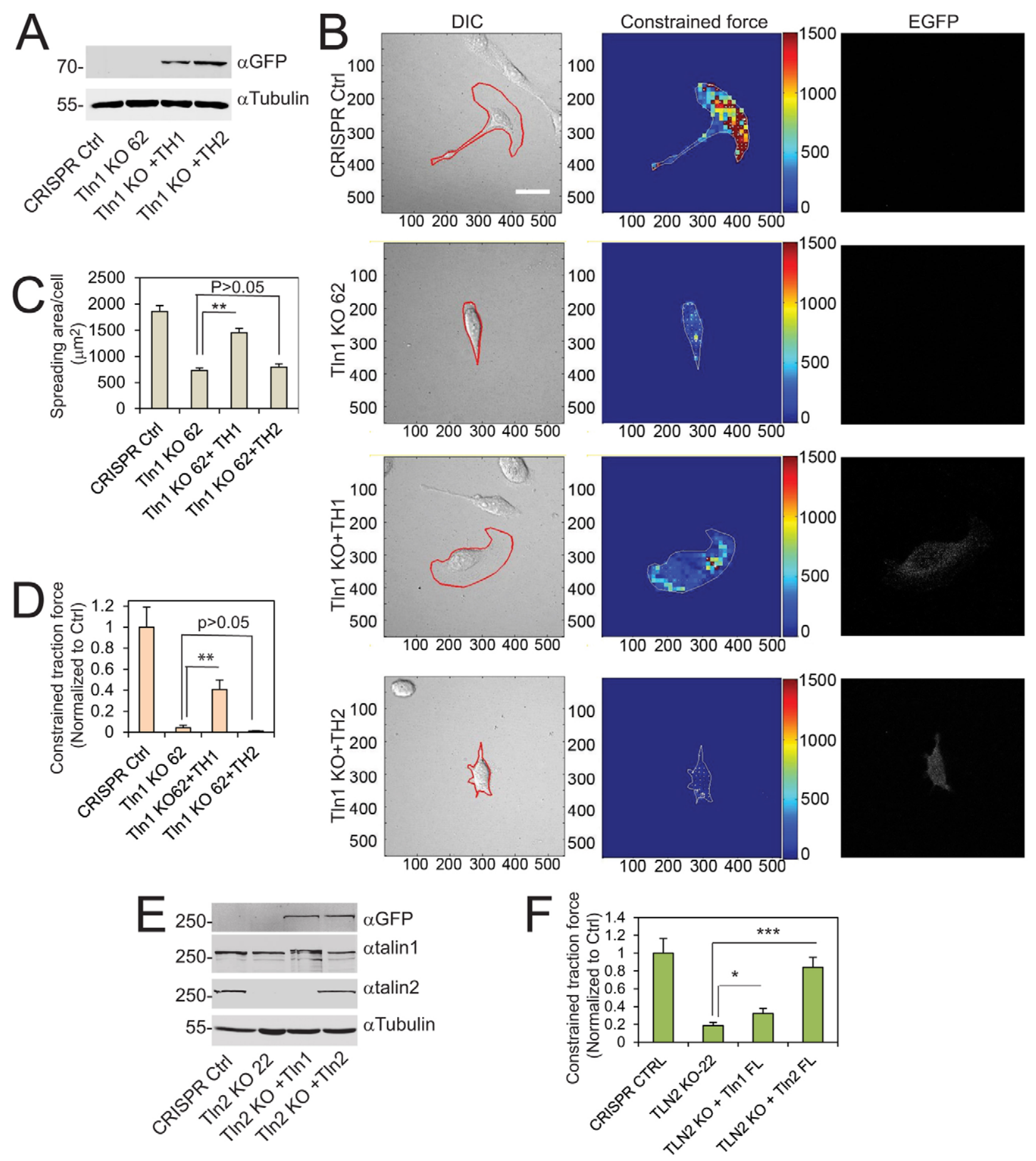

Fig. 4. Talin2 is indispensable for traction force generation. (A) Expression of EGFP-talin1 head domain (TH1) and -talin2 head domain (TH2) in talin1knockout (KO) U2OS cells. (B-D) Expression of TH1 but not TH2 partially rescued the cell-spreading and traction force defect caused by talin1 knockout.

(B) Talin1-knockout U2OS cells were transiently transfected with EGFP-TH1 or EGFP-TH2, and cultured on polyacrylamide gel containing Red FluoSpheres in order to determine the cell spreading and traction force, using CRISPR-vector-infected cells and talin1-knockout cells as controls. Axis labels are pixel numbers. Scale bar: $30 \mu \mathrm{m}$. (C) Cell spreading areas on polyacrylamide gel. Data are presented as mean \pm s.e.m. of three experiments. In each experiment, 30 cells from each group were analyzed. $t$-test, ${ }^{* *} P<0.01$. (D) Quantification of constrained traction force. Data are presented as mean \pm s.e.m. of three independent experiments. In each experiment, more than 30 cells from each group were analyzed. $t$-test, ${ }^{* *} P<0.01$. (E) Transient expression of full-length EGFP-talin 1 and EGFP-talin2 in talin2-knockout U2OS cells. (F) Re-expression of full-length EGFP-talin2 rescues the traction force defect caused by talin2 knockout, whereas reexpression of talin 1 only induced a slight increase in traction force. Data are presented as mean \pm s.e.m. of four experiments. In each experiment, more than 40 cells from each group were analyzed. $t$-test, ${ }^{*} P<0.05,{ }^{* * *} P<0.001$.

transfected with full-length EGFP-talin1 or EGFP-talin2, and the matrix degradation capabilities of these cells were determined, using talin2-knockout and CRISPR vector cells as controls.
Transfection with talin1 did not significantly improve invadopodia-mediated matrix degradation, whereas transfection with talin 2 rescued the invadopodia activity in talin2-knockout cells 

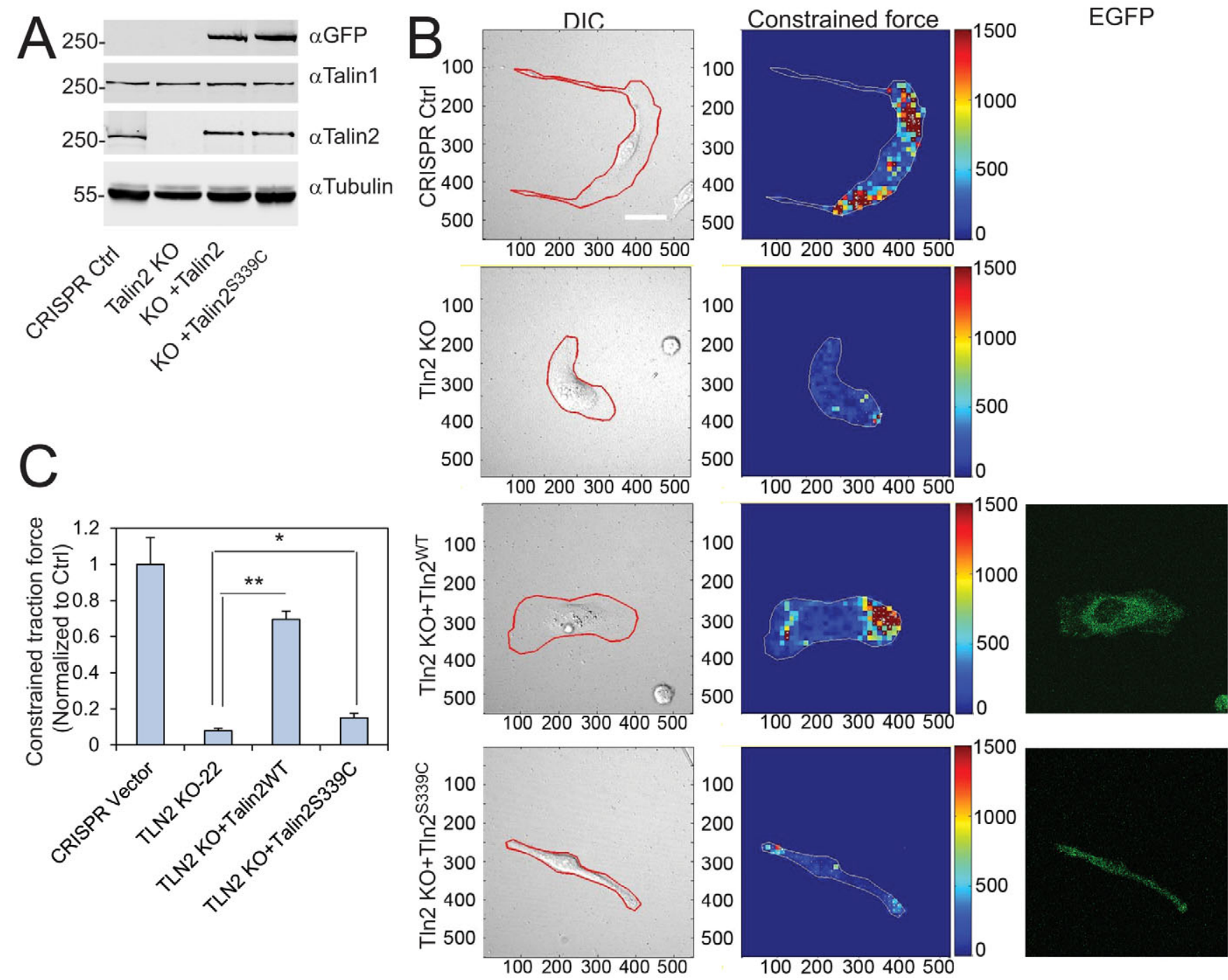

Fig. 5. Strong binding of talin2 to $\beta$-integrin tails is required for traction force generation. (A) Stable expression of EGFP-talin2 and talin2 ${ }^{\mathrm{S} 339 \mathrm{C}}$ in talin2-null (Talin2 KO) U2OS cells by using CRISPR. (B,C) Re-expression of wild-type talin2 (Talin2 ${ }^{\mathrm{WT}}$ ) in talin2-null cells restored their ability to generate traction force, but that of talin2 ${ }^{\mathrm{S} 339 \mathrm{C}}$ did not. (B) Talin2-null U2OS cells that expressed EGFP-talin2 (TIn2 ${ }^{\mathrm{WT}}$ ) or EGFP-talin2 ${ }^{\mathrm{S} 339 \mathrm{C}}$ (TIn2 ${ }^{\mathrm{S} 339 \mathrm{C}}$ ) were cultured on polyacrylamide gel containing Red FluoSpheres in order to determine traction force, using CRISPR-vector-infected cells and talin2-null cells as controls. Axis labels are pixel numbers. Scale bar: $30 \mu \mathrm{m}$. (C) Quantification of constrained traction force. Data are presented as mean \pm s.e.m. of three experiments. In each experiment, more than 30 cells from each group were analyzed. $t$-test, ${ }^{*} P<0.05,{ }^{* *} P<0.01$.

(Fig. 7F and G). Using shRNA to deplete talin2 also caused a significant reduction in invadopodium formation in U2OS cells (Fig. S4E). However, talin2-knockout did not affect the invadopodium-like pattern of cortactin staining (Fig. S4D). These results suggest that talin2 is indispensable for invadopodiummediated matrix degradation.

\section{Talin2-mediated traction force drives invadopodium- mediated matrix degradation}

To learn whether the traction force distribution is associated with invadopodia, U2OS cells were plated on Alexa-Fluor-488-gelatinconjugated polyacrylamide gels containing Red FluoSpheres. Invadopodia and traction force were measured simultaneously using a Nikon A1 confocal microscope. As shown in Fig. 8A and B, areas of bead displacement (indicating generation of traction force) were colocalized with $\sim 65 \%$ invadopodia-generated degradation holes, suggesting that traction force regulates invadopodiummediated matrix degradation.
To examine whether talin2-mediated traction force regulates invadopodium-mediated matrix degradation, talin2-null U2OS cells that stably expressed EGFP-talin2 or EGFP-talin $2^{\text {S339C }}$ were plated onto glass-bottomed dishes coated with $\mathrm{Cy} 3$-gelatin, and talin2knockout and CRISPR vector cells were used as controls. Talin2expressing cells formed invadopodium-like structures, whereas talin $2^{\mathrm{S} 339 \mathrm{C}}$-expressing cells did not (Fig. $8 \mathrm{C}$ and D). Expression of talin2 in talin2-null cells completely restored invadopodiummediated matrix degradation, whereas expression of talin $2^{\mathrm{S} 339 \mathrm{C}}$, which cannot restore the ability of cells to generate traction force, did not (Fig. 8E).

To test the role of talin2-mediated traction force in invadopodia in another cell line, talin2 expression in MDA-MB-231 cells was ablated by infecting the cells with lentiviruses that encoded Cas9 and talin2 gRNAs. AAVS1 gRNA was co-transfected with pAAVS1-EGFP-talin $2^{\mathrm{WT}}$ or pAAVS1-EGFP-talin $2^{\mathrm{S} 339 \mathrm{C}}$ into talin2-null cells. The expression of EGFP-talin2 and EGFPtalin2 ${ }^{\mathrm{S} 339 \mathrm{C}}$ in talin2-null MDA-MB-231 cells was detected using an 


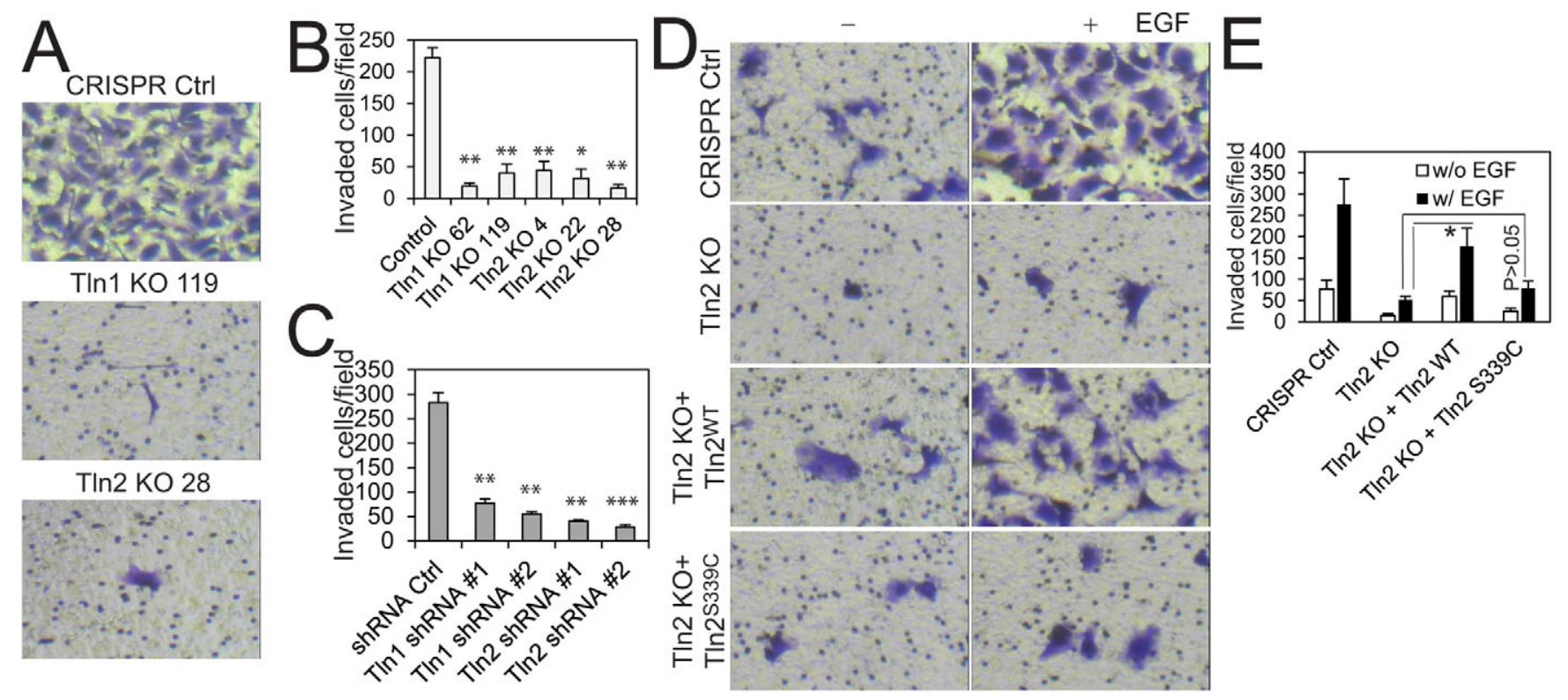

Fig. 6. Strong binding of talin2 to integrins is required for cell invasion. (A) Representative images showing that the ablation of either talin1 or talin2 (TIn1 KO and TIn2 KO, respectively) inhibited the invasion of U2OS cells. (B) Quantification of data from the experiment described in A. Data are presented as mean \pm s.e.m. from three independent experiments. $t$-test, ${ }^{*} P<0.05,{ }^{* *} P<0.01$ compared to CRISPR control cells. (C) Depletion of either talin1 or talin2 using shRNAs inhibited the invasion of MDA-MB-231 cells. Data are presented as mean \pm s.e.m. from three independent experiments. $t$-test, ${ }^{* *} P<0.01$, ${ }^{* * *} P<0.001$ compared to shRNA

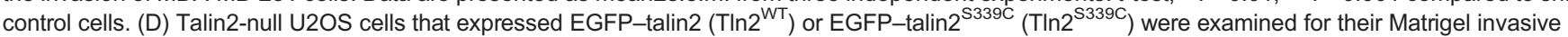
capacities, using CRISPR-vector-infected cells and talin2-null cells as controls. Representative images are shown. (E) Quantification of data from the experiment described in D. Data are presented as mean \pm s.e.m. from three independent experiments. $t$-test, ${ }^{*} P<0.05$. w/, with; w/o, without.

antibody against talin2 (Fig. 8F). Similar to the results observed in U2OS cells, talin2 markedly restored invadopodia formation in talin2-null MDA-MB231 cells, whereas talin2 ${ }^{\mathrm{S} 339 \mathrm{C}}$ had little effect (Fig. 8G and H). Thus, talin2-mediated traction force might regulate invadopodium-mediated matrix degradation.

\section{DISCUSSION}

In invasive cells, talin2 generates strong traction forces through its high affinity for $\beta$-integrins, driving invadopodium-mediated matrix degradation and ultimately, cell invasion (Fig. 8I). Thus, talin2 binding to $\beta$-integrin tails is indispensable for cancer cell invasion.

We found that talin 2 binds to integrin tails stronger than talin 1 does and that the talin2 residue S339 contributes to its strong interaction with $\beta$-integrins. Substitution of S339 with Cys caused a reduction in its binding to integrins, whereas the reciprocal mutation of talin1 C336 to Ser enhanced talin1 binding (Fig. 1). Our data are different from the results of a previous report (Anthis et al., 2009), in which the F3 FERM domains of talin 1 and talin2 were found to bind to $\beta 1 \mathrm{~A}$-integrin tails with a $K_{\mathrm{d}}$ of 491 and $652 \mu \mathrm{M}$, respectively, as measured by using nuclear magnetic resonance NMR. In our study, we found that talin 2 had a stronger interaction with $\beta$-integrin tails than talin1; this discrepancy is probably because we used the fulllength talin 2 head domain, whereas the F3 domain alone was used by Anthis et al. (2009). Indeed, the talin 2 head domain bound to B1A-integrin tails much more efficiently than the F2-F3 FERM domain (Fig. S1C). This is consistent with a previous report (Calderwood et al., 1999). The discrepancy could be also caused by the different buffers used in the assays, where we included $3 \mathrm{mg} / \mathrm{ml}$ BSA or $0.5 \mathrm{mg} / \mathrm{ml}$ gelatin in our binding buffer to reduce nonspecific binding. Our findings provide a molecular basis to explain the different roles of talin1 and talin2.

Talin1 and talin2 have distinct roles in regulating focal adhesion assembly. Based on staining of FAK and zyxin, knockout or knockdown of talin1 inhibited the formation of small focal adhesions $\left(<7 \mu \mathrm{m}^{2}\right)$, but had no effect on large focal adhesions $\left(>7 \mu \mathrm{m}^{2}\right)$ (Fig. 2; Fig. S2). Knockout or knockdown of talin2 also suppressed zyxin localization to focal adhesions but did not influence the recruitment of FAK to focal adhesions. These results are consistent with previous findings obtained in talin1-knockout cells (Zhang et al., 2008). Because Zyxin is a marker for focal adhesion maturation (Zaidel-Bar et al., 2003), these results suggest that talin1 regulates small focal adhesion assembly, whereas talin2 controls larger, more stable focal adhesion formation. Talin1mediated smaller focal adhesions might contribute to focal adhesion dynamics and cell migration (Huang et al., 2009), whereas the ability of talin2 to aggregate into large assemblies might stabilize invadopodia.

We found that talin2 plays an important role in traction force generation. Knockout or knockdown of talin2 suppressed traction force generation (Fig. 3; Fig. S3). Moreover, the traction force in talin2-knockout cells can be rescued by transfecting cells with talin2, but not with talin1, indicating the indispensable role of talin2 in traction force generation (Fig. 4). Furthermore, talin2-mediated traction force is dependent on its strong interaction with integrin tails (Fig. 5). Talin2-mediated traction force generation could be related to the role of talin 2 in large focal adhesion formation, because focal adhesions regulate traction force generation (Dumbauld et al., 2013; Hinz and Gabbiani, 2003; Morimatsu et al., 2015). Ablation of talin1 caused a reduction in traction force, but this effect can be partially attributed to the role of talin 1 in cell spreading (Figs 3 and 4) because: (1) knockout of talin1 also inhibited cell spreading (Fig. 3), and (2) expression of the talin1 head domain in talin1-null cells promoted spreading and traction force generation (Fig. 4). The talin1 head domain retains the integrin-activating function of talin 1 , thus promoting cell spreading (Calderwood et al., 1999; Zhang et al., 2008). However, it lacks the 

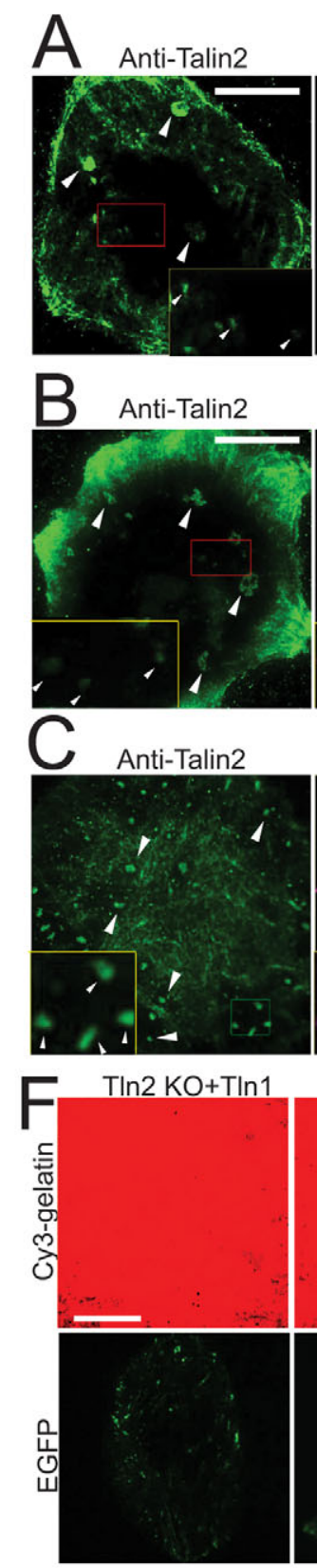

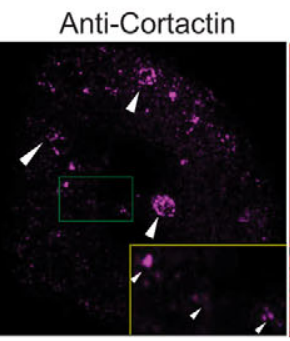

Anti-Tks5

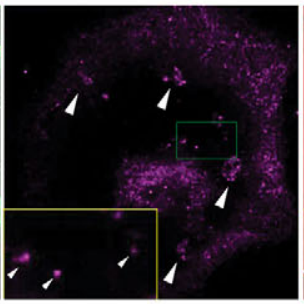

Anti-Cortactin

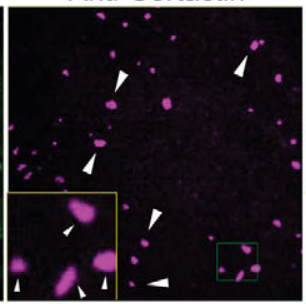

$\mathrm{T} \ln 2 \mathrm{KO}+\mathrm{T} \ln 2$

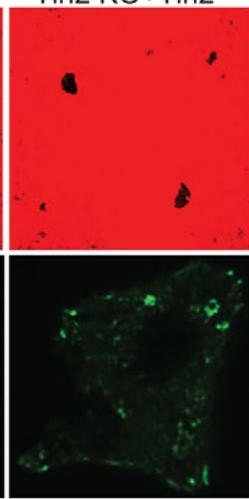

Cy3-gelatin

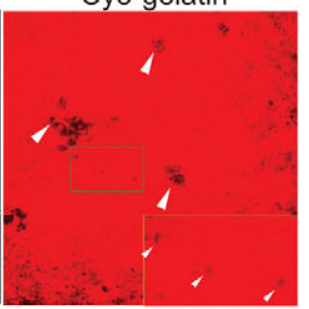

Cy3-gelatin

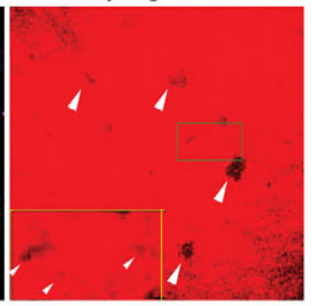

Cy3-gelatin
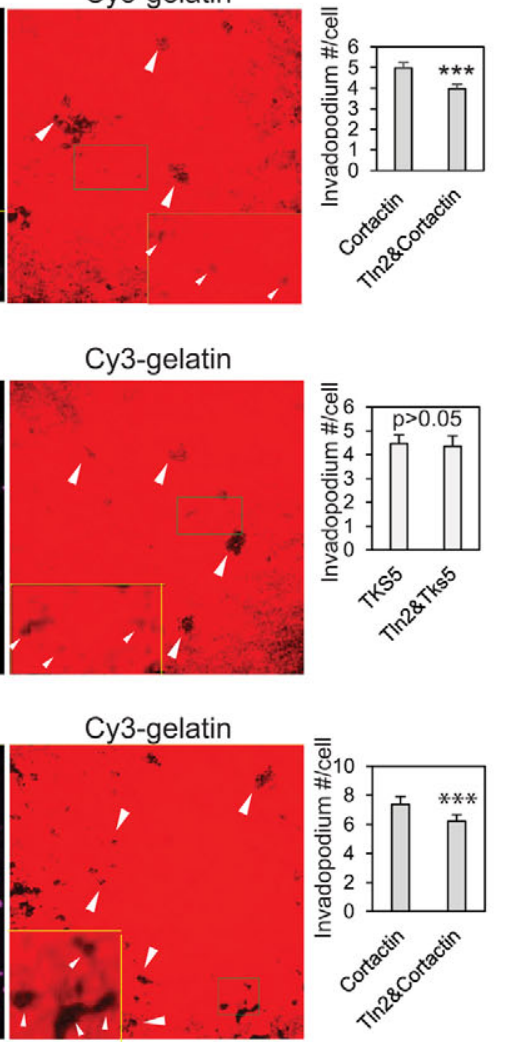

$\mathrm{D}$
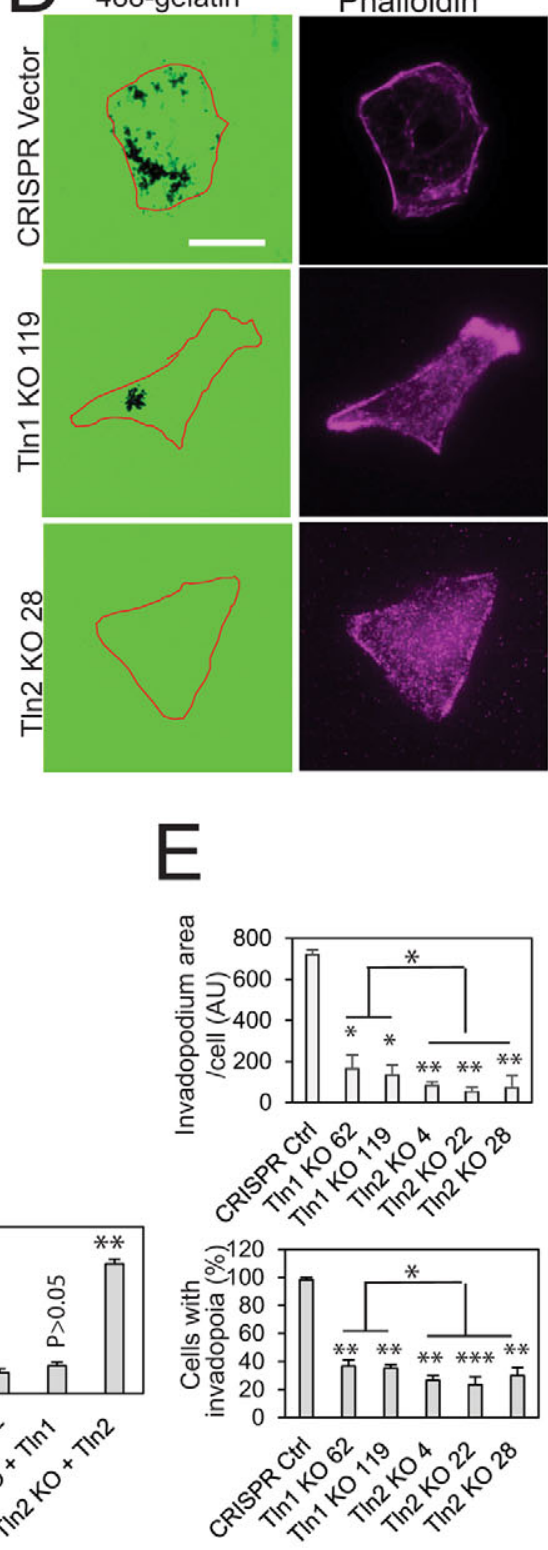

Fig. 7. Talin2 is indispensable for invadopodium-mediated matrix degradation. (A,B) Talin2 colocalized with cortactin (A) and Tks5 (B) at invadopodia in U2OS cells plated on Cy3-labeled gelatin for $16 \mathrm{~h}$. Cells were co-stained with chicken anti-talin2 and rabbit anti-cortactin antibodies (A), or with anti-talin2 and anti-Tks5 (B) antibodies. Representative TIRF images are shown. Arrowheads point to mature invadopodia in which talin2 colocalized with cortactin (A) or Tks5 (B). Insets show magnified images of small invadopodia in the box. Scale bars: $20 \mu \mathrm{m}$. Graphs show the mean number of degradation spots with cortactin (left bar) and with both cortactin and talin2 (right bar) (A), or with Tks5 (left bar) and with both Tks5 and talin2 (right bar) (B). Data are presented as mean \pm s.e.m. of 40 cells from two independent experiments. (C) Talin2 colocalized with cortactin at invadopodia in MDA-MB-231 cells plated on Cy3-labeled gelatin for $10 \mathrm{~h}$. $t$-test, ${ }^{* * *} P<0.001$. (D) Ablation of either talin1 or talin2 inhibited invadopodium-mediated matrix degradation. Talin1- or talin2-null (TIn1 KO 119 and TIn2 KO 28 , respectively) U2OS cells were cultured on Alexa-Fluor-488-gelatin immobilized on glass-bottomed dishes, fixed and stained with Alexa-Fluor-647-phalloidin. Scale bar: $20 \mu \mathrm{m}$. (E) Quantification of data from the experiment described in D. Data are presented as mean \pm s.e.m. of three independent experiments. In each experiment, 20 cells from each group were analyzed. $t$-test, ${ }^{*} P<0.05,{ }^{* *} P<0.01,{ }^{* * *} P<0.001$. (F) Re-expression of full-length EGFP-talin2, but not of EGFP-talin1, restored invadopodial matrix degradation in talin2-null (TIn2-KO) cells. Talin2-knockout U2OS cells were transiently transfected with full-length EGFP-talin1 or EGFP-talin2, and cultured on Cy3-gelatin immobilized on glass-bottomed dishes, fixed and stained with Alexa-Fluor-647-phalloidin, using CRISPR-vectorinfected cells and talin2-knockout cells as controls. Scale bar: $20 \mu \mathrm{m}$. (G) Quantification of the data from the experiment described in F. Data are presented as mean \pm s.e.m. of three independent experiments. $t$-test, ${ }^{* *} P<0.01$. Representative images are shown.

major actin binding site and is deficient in mediating traction force (Zhang et al., 2008). The distinct roles of talin1 and talin2 in cell spreading and traction force could be caused by their different binding affinities toward $\beta$-integrins. This assertion is supported by the deficiency of talin $2^{\mathrm{S} 339 \mathrm{C}}$ in mediating traction force (Fig. 5). It is possible that the different mechanical properties between talin 1 and talin2 rod domains could contribute to traction force generation (Austen et al., 2015). Taken together, it is likely that 

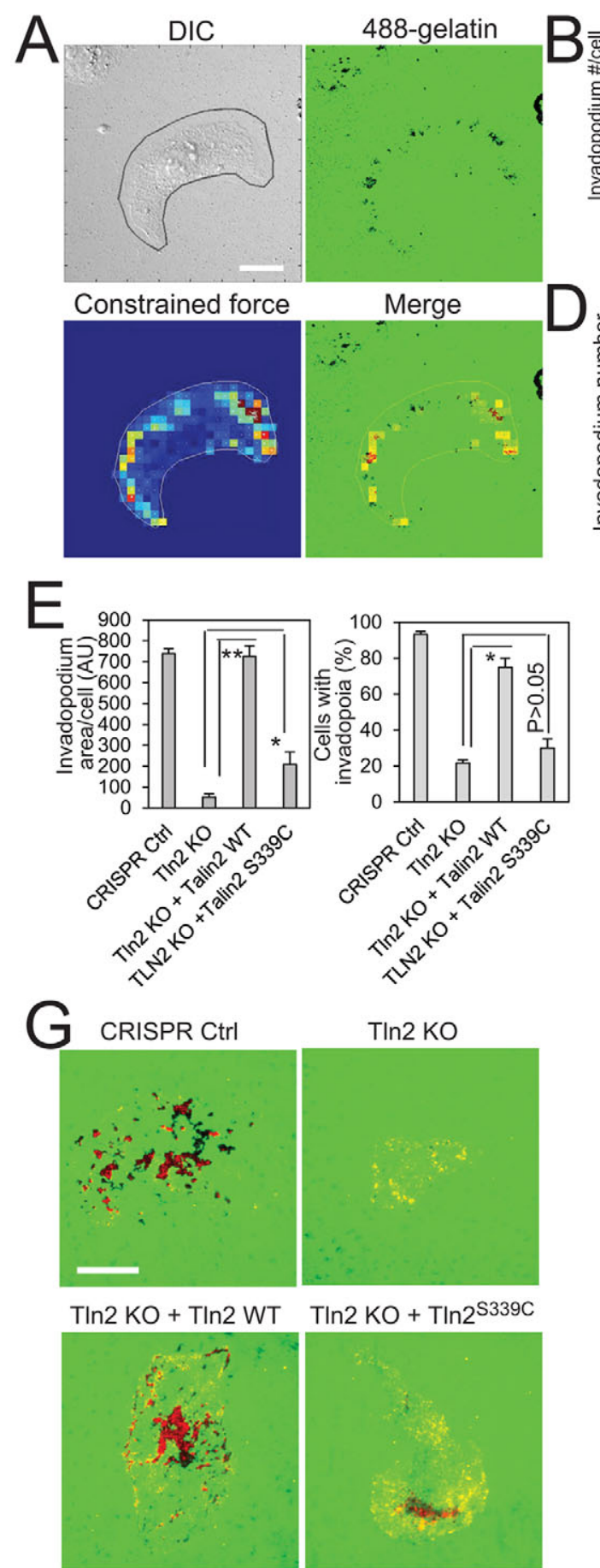

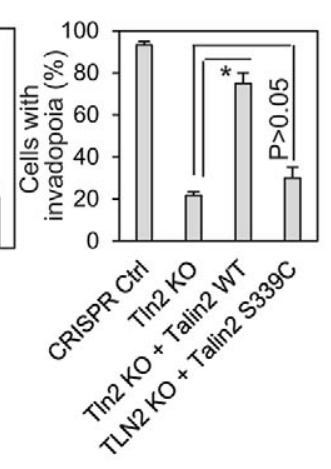

TIn2 KO

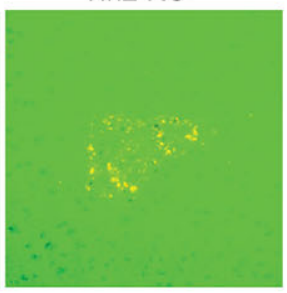

$\mathrm{T} \ln 2 \mathrm{KO}+\mathrm{T} \ln 2^{\mathrm{S} 339 \mathrm{C}}$

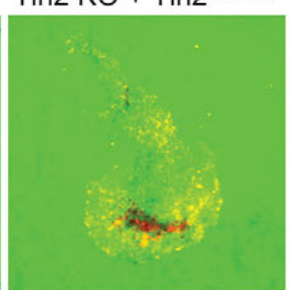

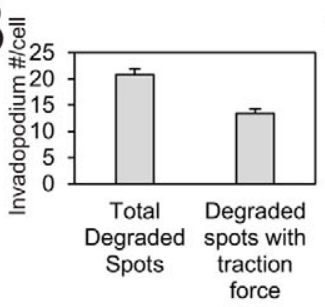
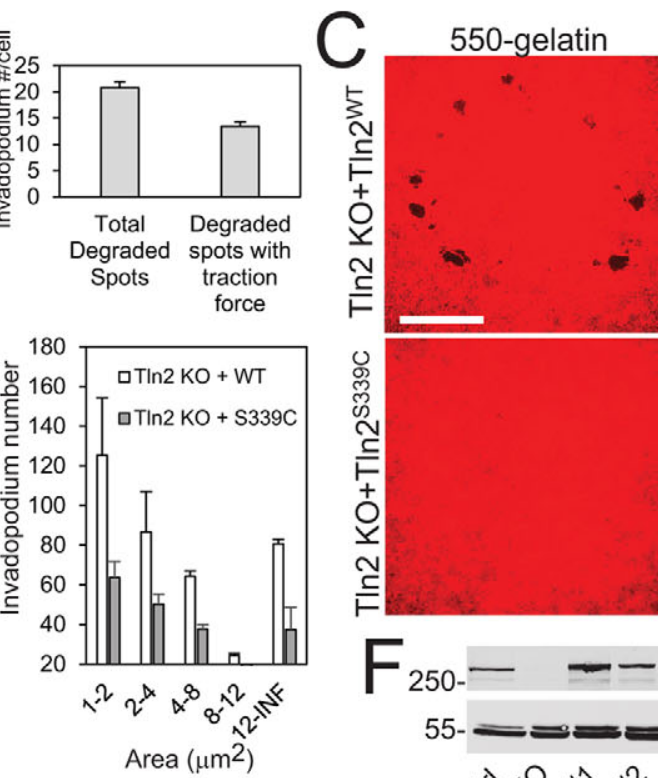
Anti-talin2
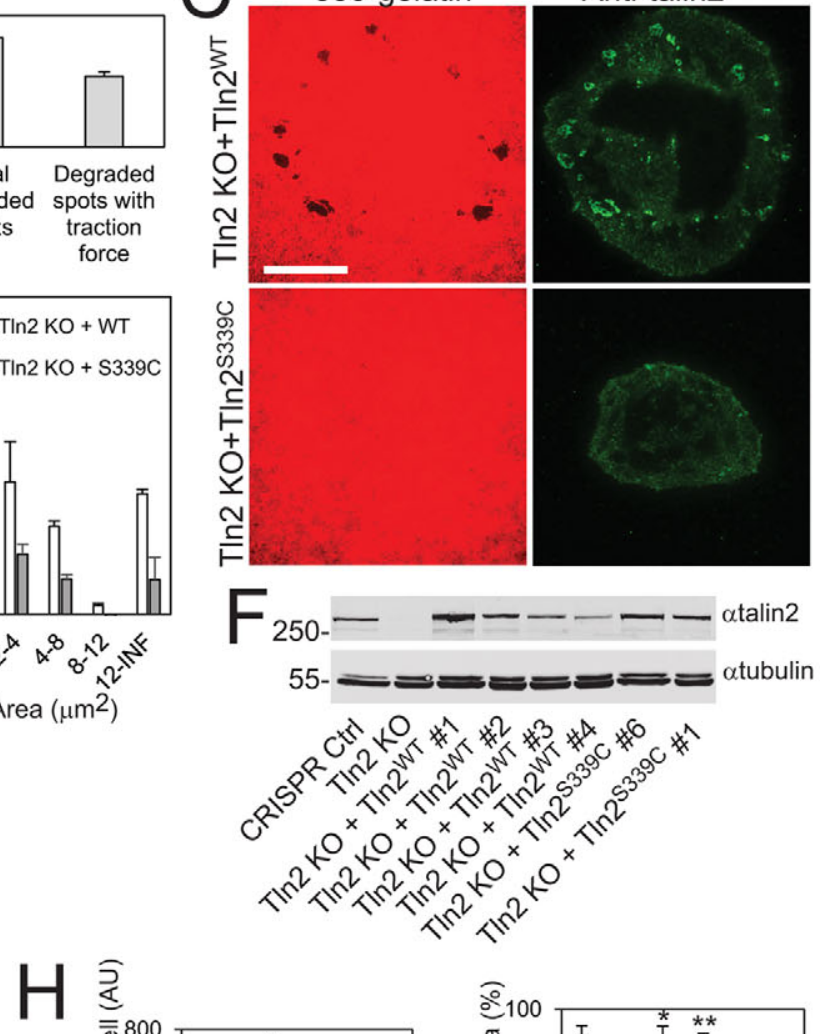

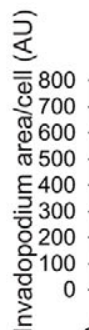

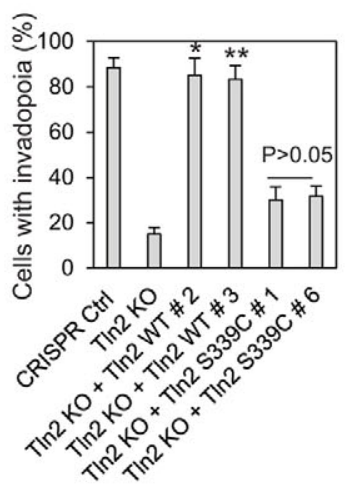

T

Talin2

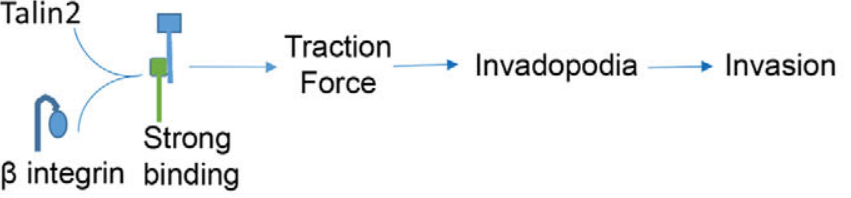

Fig. 8. Strong binding of talin2 to $\beta$-integrin tails is indispensable for invadopodium-mediated matrix degradation. (A) The distribution of traction force was associated with invadopodia. USOS cells were cultured on Alexa-Fluor-488-gelatin immobilized on acrylamide gel containing Red FluoSpheres. Traction force and invadopodium formation were determined using an A1 confocal microscope. Scale bar: $30 \mu \mathrm{m}$. (B) Quantification of the association of traction force and invadopodia. The gelatin degradation images were merged with traction force maps, and colocalization was examined manually. Data are presented as mean \pm s.e.m. of 24 cells from three independent experiments. (C-E) Stable expression of EGFP-talin2 (Talin2 WT) in talin2-null (TIn2 KO) U2OS cells restored invadopodial matrix degradation, but that of EGFP-talin2 ${ }^{\mathrm{S} 339 \mathrm{C}}$ did not. (C) Talin2-null U2OS cells that stably expressed EGFP-talin2 or EGFP-talin2 ${ }^{\mathrm{S} 339 \mathrm{C}}$ were cultured on Cy3-gelatin immobilized on glass-bottomed dishes, fixed and stained for talin2. Scale bar: $20 \mu \mathrm{m}$. (D) Quantification of the assembly of talin2 and talin2 ${ }^{\text {S339C }}$ in invadopodium-like structures. Data are presented as mean \pm s.e.m. of three independent experiments. (E) Quantification of invadopodium area. Data are presented as mean \pm s.e.m. of three independent experiments. In each experiment, 20 cells from each group were analyzed. $t$-test, ${ }^{*} P<0.05,{ }^{*} P<0.01$. $(\mathrm{F}-\mathrm{H})$ Talin2-null MDA-MB-231 cells that expressed EGFP-talin2 (TIn2 ${ }^{\mathrm{WT}}$ ) or -talin2 ${ }^{\mathrm{S} 339 \mathrm{C}}$ were cultured on Alexa-Fluor-488-gelatin immobilized on

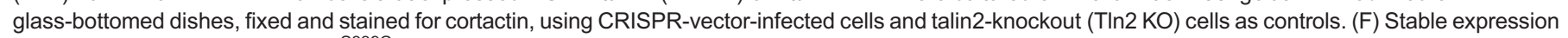
of EGFP-talin2 and EGFP-talin2 ${ }^{\text {S339C }}$ in talin2-null MDA-MB-231 cells using CRISPR. The numbers after the cells and constructs denote different clones.

(G) Merged TIRF images of matrix degradation and cortactin staining. Scale bar: $20 \mu \mathrm{m}$. (H) Expression of EGFP-talin2 in talin2-null MDA-MB-231 cells restored invadopodial matrix degradation, but that of EGFP-talin $2^{\mathrm{S} 339 \mathrm{C}}$ did not. Data are presented as mean \pm s.e.m. of three independent experiments. $t$-test, ${ }^{*} P<0.05$, ${ }^{* \star} P<0.01,{ }^{* \star *} P<0.001$, compared to talin $2 \mathrm{KO}$ cells. (I) A proposed mechanism by which talin2 regulates traction force generation, invadopodium-mediated matrix degradation and cancer cell invasion. 
talin 1 indirectly modulates traction force through promoting cell spreading, whereas talin 2 might directly control traction force by acting as a mechanical transmitter between integrins and the actin cytoskeleton.

Although it has been reported that talin2 is not essential for fibroblast migration (Debrand et al., 2012), we demonstrate that it plays an important role in cell invasion. Knockout or knockdown of talin2 inhibited cancer cell invasion (Fig. 6). Furthermore, reexpression of wild-type talin2 in talin2-null cells rescued cell invasion, whereas re-expression of talin $2^{\mathrm{S} 339 \mathrm{C}}$, which is deficient in binding to $\beta$-integrin, did not (Fig. 6). The role of talin2 in cell invasion can be attributed to its effect on invadopodium-mediated matrix degradation, a key regulatory point for cell invasion (Beaty and Condeelis, 2014; Bergman et al., 2014; Paz et al., 2014; Weaver, 2006; Yamaguchi et al., 2006).

Invadopodia are large assemblies that mediate cell invasion (Beaty and Condeelis, 2014; Paz et al., 2014; Revach and Geiger, 2014). We found that talin 2 colocalized with invadopodia to a considerable extent (Fig. 7). In fact, large and round talin2 staining spots usually colocalized with invadopodia. Ablation of either talin1 or talin2 reduced invadopodium-mediated matrix degradation, but ablation of talin 2 had slightly more severe effects (Fig. 7). Furthermore, expression of full-length wild-type talin2 rescued invadopodium-mediated matrix degradation in talin2-null cells, whereas expression of talin $2^{\mathrm{S} 339 \mathrm{C}}$, which has reduced binding to $\beta 1$-integrin tails, had little effect (Fig. 8). These results suggest that a strong interaction between talin 2 and $\beta$-integrin tails is indispensable for invadopodium-mediated matrix degradation.

Interestingly, regions of high traction forces also coincided with invadopodia (Fig. 8A). Re-expression of wild-type talin2 but not talin $2^{\mathrm{S} 339 \mathrm{C}}$ in talin2-null cells restored the traction force generation and invadopodium-mediated matrix degradation (Fig. 5; Fig. 8). These results suggest that binding of talin 2 to $\beta$-integrin tails is essential for the generation of traction force, which in turn drives invadopodium-mediated matrix degradation and cancer cell invasion.

It has been reported that podosomes, which are similar structures to invadopodia, develop in the absence of traction forces (Yu et al., 2013). We found here that talin2-mediated traction force was required for invadopodium-mediated matrix degradation. However, talin2 does not seem to be required for the initial development of invadopodia because knockout of talin2 did not influence the assembly of cortactin, an invadopodium marker, into invadopodium-like structures (Fig. S4). Thus, talin2-mediated traction force might instead regulate invadopodium stability or maturation. Although we still do not understand how traction force regulates invadopodia, our findings fill in the gaps in our existing knowledge of talin2 and uncover new but fundamental roles of talin2 in mediating traction force and invadopodium development during cell invasion.

Talin 1 is generally thought to play more important roles than talin 2 in cell migration and embryogenesis - talin1 knockout causes embryonic lethality in mice (Monkley et al., 2000), whereas talin2 is not essential for mouse embryonic development (Debrand et al., 2012). However, as we show here, talin 2 generates traction force to mediate invadopodium formation and cell invasion. The recent identification of human pathology - the digit deformity fifth finger camptodactyly - arising from a missense variant, S339L, of the TLN2 gene (Deng et al., 2016) demonstrates the physiological importance of residue S339 in talin2 function and the requirement for strong talin2-integrin linkage for normal development. Both an S339C and the disease-copying S339L mutant reduced the affinity of talin 2 for integrin and the ability of cells to generate traction forces. It is possible that the deleterious effect of this mutation on integrin binding and traction force generation could be the cause of the developmental abnormality. Interestingly, talin2 has been found to be downregulated by trastuzumab, a HER2-targeting antibody drug for treatment of breast cancers (Le et al., 2012). Thus, inhibition of talin 2 function could be a potential strategy for cancer therapy.

\section{MATERIALS AND METHODS \\ Reagents}

Anti-talin1 (clone 97H6) and anti-talin2 (clone 53.8) antibodies were from AbD Serotec. Anti-zyxin (clone EPR4302) rabbit monoclonal antibody was from Abcam. Anti-cortactin mouse monoclonal antibody (clone 4F11) and anti-Tks5 rabbit polyclonal antibody (clone SH3 \#4) were from EMD Millipore. Anti-cortactin (clone H222) rabbit polyclonal and anti-N-WASP (clone 30D10) rabbit monoclonal antibodies were from Cell Signaling Technology. Anti- $\beta 1$-integrin monoclonal antibody (clone P5D2) was from R\&D Systems. Anti-phosphorylated-FAK (at Y397) [clone 18/FAK (pY397)] was from BD Biosciences. Anti-talin2 (clone GW22654) chicken polyclonal and anti-tubulin (clone B-5-1-2) monoclonal antibodies, bovine skin gelatin and pLKO1 lentivirus shRNAs that targeted talin1 and talin2 were from Sigma-Aldrich. For western blotting, anti-tubulin antibody was used at 1:5000 dilution, all other antibodies at 1:1000 dilution. For immunofluorescence staining, anti-zyxin and antiphosphorylated-FAK antibodies were used at 1:300 dilution, all other antibodies were used at 1:100. Talin1 shRNA clones were TRCN0000123105 (\#1) and TRCN0000299020 (\#2). Talin2 shRNA clones were TRCN0000122990 (\#1) and TRCN0000122992 (\#2). LentiCRISPRv2 and pSpCas9(BB)-2A-Puro V2.0, which were generated by Feng Zhang's laboratory (Ran et al., 2013), were from Addgene. AlexaFluor-488-labeled gelatin and Red FluoSpheres were from Life Technologies. Cy3 dye was from Click Chemistry Tools. Gelatin was labeled with $\mathrm{Cy} 3$ according to the manufacturer's instruction. DyLight-488conjugated goat anti-mouse IgG $(\mathrm{H}+\mathrm{L})$ and Alexa-Fluor-488-labeled goat anti-chicken $\operatorname{IgY}(\mathrm{H}+\mathrm{L})$ were from Thermo Scientific. Dylight-550- or -633labeled goat anti-mouse and anti-rabbit $\operatorname{IgG}(\mathrm{H}+\mathrm{L})$ were from Immunoreagents (Raleigh, NC). Bovine fibronectin and recombinant human EGF were from Akron Biotech; growth-factor-reduced Matrigel was from BD Bioscience. Pfu Ultra was from Agilent Technologies. Cold Fusion Cloning Kit was from System Biosciences (Palo Alto, CA). AntiGFP monoclonal antibody and Safectine RU50 transfection kit were purchased from Syd Labs (Malden, MA). DNA primers were synthesized by Integrated DNA Technologies.

\section{Plasmid construction}

The full-length pEGFP-talin2 plasmid encoding the wild-type protein was subcloned using the following steps: (1) DNA fragments encoding residues 1-1159 of human talin2 were amplified by using Pfu-Ultra-based PCR and the human talin2 cDNA clone as the template and 5'-atgcactcgagctatggtggecctgtccttaaagatttgt-3' and 5'-actgaggtaccgtctcgagcagaatctaacatggcat- $3^{\prime}$ as primers, the product was then subcloned into pEGFP-C1 with the Xho1 and Kpn1 sites; (2) fragments encoding residues 1160-2543 of talin2 were amplified using human cDNA from U2OS cells and $5^{\prime}$-ggctgcatcgacaaccgacccc-3' and 5'-tattatctagattagccetcatcttccetcagctc- $3^{\prime}$ and subcloned into the plasmid generated in step 1 into the Not1 and Xba1 sites. pEGFPtalin $2_{1-449}$ was generated by amplifying DNA fragments encoding residues 1-449 using 5'-ATGCACTCGAGCTATGGTGGCCCTGTCCTTAAAGATTTGT-3' and 5'-GGGCCCGTCGACTATGAGCCGTGCTCTGCCTTCCC-3' as primers, and subcloning into pEGFP-C1 vector through Xho1 and Sal1 sites. pEGFP-talin $1_{1-446}$ was generated by amplifying DNA fragments encoding residues 1-446 using 5'-GGGCCCGAATTCTATGGTTGCACTTTCACTGAAGATCAG-3' and 5'-GGGCCCGTCGACTTAAGAGCCATGCTCCACTTTCCCC-3' as primers, and subcloning into pEGFP-C1 vector into the EcoR1 and Sal1 sites. pEGFP-talin $2_{1-449}$ S339C was created by performing Pfu-Ultra-based PCR using pEGFP-talin $2_{1-449}$ as template and 5'-GGATCACCAAAGACTGTGTGATGCGCGTGG-3' 
and 5'-CCACGCGCATCACACAGTCTTTGGTGATCC-3' as primers. pEGFP-talin $1_{1-446}{ }^{\mathrm{C} 336 \mathrm{~S}}$ was created by performing PCR using pEGFPtalin $1_{1-446}$ as template and $5^{\prime}$-CATCACCAAGGAGAGTGTGATGCGAG$3^{\prime}$ and $5^{\prime}$-CTCGCATCACACTCTCCTTGGTGATG-3' as primers. pEGFPtalin $1_{1-433}$ has been described previously (Huang et al., 2009). pQEtalin $1_{1-446}$ and pQE-talin $2_{1-449}$ were generated by amplifying the DNA fragments using 5'-GGGCCCGAGCTCATGGTTGCACTTTCACTGAAGATCAG-3' and 5'-GGGCCCGTCGACTTAAGAGCCATGCTCCACTTTCCCC-3', and 5'-ATGCAGAATCCATGGTGGCCCTGTCCTTAAAGATTTGT-3' and 5'-GGGCCCGTCGACTATGAGCCGTGCTCTGCCTTCCC- $3^{\prime}$ as primers and subcloning into pQE-30 vector into the Sac1 and Sal1 sites, and the BamH1 and Sall sites, respectively. The rescue plasmids pEGFP-talin $2_{1-449}-\mathrm{R}$ and pEGFP-talin $2_{1-449}{ }^{\mathrm{S} 339 \mathrm{C}}-\mathrm{R}$ were created by performing PCR using pEGFP-talin $2_{1-449}$ as template and $5^{\prime}$-GTGAAGACCATGCAGTTCGAGCCATCTACAGCTGT-3' and 5'-ACAGCTGTAGATGGCTCGAACTGCATGGTCTTCAC- $3^{\prime}$ as primers. The full-length rescue plasmids pEGFP-talin2-R and pEGFP-talin $2^{\mathrm{S} 339 \mathrm{C}}-\mathrm{R}$ were created by digesting full-length pEGFP-talin2 with BsrG1 and EcoRV, and ligating the resulting larger fragment with the smaller fragments from the rescue plasmids pEGFP-talin $2_{1-449}-\mathrm{R}$ and pEGFPtalin2 ${ }_{1-449}{ }^{\mathrm{S} 339 \mathrm{C}}$-R. The full-length pAAVS1-EGFP-talin2 ${ }^{\mathrm{WT}}$ and pAAVS1EGFP-talin2 ${ }^{\mathrm{S} 339 \mathrm{C}}$ plasmids were created by subcloning talin2 and the mutant into pAAVS1-EGFP vector, using the same strategy as used to subclone full-length pEGFP-talin2. The pAAVS1-EGFP vector was generated by following the following procedures: (1) pEGFP-C1 with BsiWI (at nucleotide 20) and AscI sites (at nucleotide 3533) was created by sequential PCR using pEGFP-C1 as template and 5'-GTTATTAATAGTAATCACGTACGGGGTCATTAGTTCATAG-3' and 5'-CT-ATGAACTAATGACCCCGTACGTGATTACTATTAATAAC$3^{\prime}$ and $5^{\prime}$-CGGAATCGTTTTCCGGCGCGCCGGCTGGATGATC-3' and 5'-GATCATCCAGCCGGCGCGCCGGAAAACGATTCCG-3' as primers; (2) the left and right homologous arms of AAVS1 were amplified by performing PCR using $5^{\prime}$-TTAATAGTAATCACGTACGTGCTTTCTCTGACCAGCATTC-3' and 5'-ATGAACTAATGACCCCGTACGGCCCCACTGTGGGGTGGA-3', and 5'-CGGAATCGTTTTCCGGCGCGCCACTAGGGACAGGATTGGTG-3' and 5'-GGATCATCCAGCCGGCGCGCCAGAGCAGAGCCAGGAACCC-3' as primers, respectively, and the products were subcloned into the modified $\mathrm{pEGFP-C1}$ vector using the BsiWI and AscI sites, respectively, using the Cold Fusion Cloning Kit. AAVS1 gRNA was generated by annealing oligos 5'-CACCGCTAGTGGCCCCACTGTGGGG-3' and 5'-AAACCCCCACAGTGGGGCCACTAGC-3', and subcloning into BbsI-digested pSpCas9(BB)-2A-Puro V2.0 vector. LentiCRISPR-TLN1 was created by annealing oligos 5'-CACCGGGATCCGCTCACGAATGATG-3' and 5'-AAACCATCATTCGTGAGCGGATCCC-3', and subcloning into BsmB1-digested lentiCRISPRv2 vector. LentiCRISPR-TLN2 was generated by annealing oligos 5'-CACCGCGTGTCGAGTCATTCGGGAA-3' and 5'-AAACTTCCCGAATGACTCGACACGC-3', and subcloning into lentiCRISPRv2 vector. All plasmids were sequenced by Eurofins MWG Operon (Huntsville, AL).

\section{Cell culture and transfection}

CHO-K1 Chinese hamster ovary cells, MDA-MB-231 human breast cancer cells, U2OS human bone osteosarcoma cells and $293 \mathrm{~T}$ human embryonic kidney cells were from the American Type Culture Collection, and were maintained in Dulbecco's modified Eagle medium (DMEM; Corning Inc.) containing $10 \%$ fetal bovine serum (FBS), penicillin $(100 \mathrm{U} / \mathrm{ml})$ and streptomycin $(100 \mu \mathrm{g} / \mathrm{ml})$. CHO-K1 and 293T cells were transfected using Safectine RU50 according to the manufacturer's protocol. U2OS cells were transfected using Mirus Ingenio solution using GenePulser Xcell (Bio-Rad Inc., Hercules, CA).

\section{Knockout of TLN1 and TLN2 with CRISPR}

Preparation of viruses and cell infection were performed as described previously (Li et al., 2013; Wu et al., 2011). LentiCRISPR-TLN1 and lentiCRISPR-TLN2 were co-transfected with packaging vectors $\mathrm{pMDLg} / \mathrm{pRRE}$, pRSV-Rev and CMV-VSVG into 293FT cells. Lentiviral particles were collected and used to infect U2OS cells. The cells were selected with puromycin, and clones were isolated. TLN1- or TLN2-knockout clones were detected by western blotting using anti-talin 1 and anti-talin2 monoclonal antibodies.

\section{Re-expression of talin2 and talin2 ${ }^{\mathrm{S339C}}$ in talin2-null cells}

The AAVS1 gRNA was co-transfected with pAAVS1-EGFP-talin2 ${ }^{\mathrm{WT}}$ or -EGFP-talin2 ${ }^{\mathrm{S} 339 \mathrm{C}}$ into talin2-null cells. Transfected cells were selected with neomycin. EGFP-positive cells were sorted by using flow cytometry, or EGFP-positive clones were isolated by using cloning cylinders.

\section{Protein interaction assays}

CHO-K1 cells were transfected with pEGFP-talin $1_{1-433}$, - talin $1_{1-446},-$ talin $1_{1-}$ 449 , or their mutants. At $28 \mathrm{~h}$ post transfection, the cells were harvested in lysis buffer A (50 mM Tris-HCl pH 7.4, 1\% NP-40, 150 mM NaCl, 1 mM EDTA and a protease inhibitor cocktail). Cell lysates were cleared by centrifugation and incubated with glutathione-Sepharose beads loaded with GST or GST- $\beta$ integrin tails at $4^{\circ} \mathrm{C}$ for $2 \mathrm{~h}$. The beads were washed with the lysis buffer four times and resuspended in SDS sample buffer. Samples were analyzed using SDS-PAGE and transferred to nitrocellulose membrane for the detection of interacting proteins. The binding of purified His-tagged proteins to GST- $\beta$ integrin tails was performed in lysis buffer A containing $3 \mathrm{mg} / \mathrm{ml} \mathrm{BSA}$ (Fig. 1D) or $0.5 \mathrm{mg} / \mathrm{ml}$ gelatin (Fig. 1I).

\section{Cell invasion assays}

Cell invasion was performed as described previously (Li et al., 2013). Briefly, $100 \mu \mathrm{l}$ of Matrigel (1:30 dilution in serum-free DMEM) was added to each Transwell polycarbonate filter and incubated at $37^{\circ} \mathrm{C}$ for $5 \mathrm{~h}$. Cells were trypsinized and washed three times with DMEM containing 1\% FBS. The cells were resuspended in DMEM containing $1 \%$ FBS at a density of $5 \times 10^{5}$ cells $/ \mathrm{ml}$. The cell suspensions $(100 \mu \mathrm{l})$ were seeded into the upper chambers, and $600 \mu \mathrm{l}$ of DMEM containing 1\% FBS, $20 \mathrm{ng} / \mathrm{ml}$ EGF and $10 \mu \mathrm{g} / \mathrm{ml}$ fibronectin was added to the lower chambers. The cells were allowed to invade for $12 \mathrm{~h}$ (or as indicated) in a $\mathrm{CO}_{2}$ incubator, fixed, stained and quantified as described previously (Wu et al., 2011).

\section{Traction force measurement}

Glass-bottomed dishes were silanized with $0.5 \%$ silane, and activated with $0.5 \%$ glutaraldehyde. A drop of gel solution containing acrylamide $(6 \%)$, bis-acrylamide $(0.75 \%)$, ammonium persulfate (APS), TEMED and FluoSpheres ${ }^{\circledR}$ carboxylate-modified beads (diameter $0.2 \mu \mathrm{m}, 1: 85$ dilution by volume) was added to the dishes and covered by a coverslip. The coverslip was removed, and gels were activated with sulfo-SANPAH under UVA light and then conjugated with gelatin $(0.2 \mathrm{mg} / \mathrm{ml})$. Cells were plated onto the gels and traction force was measured as described previously (Butler et al., 2002), using an A1 confocal microscope in Lexington VA Medical Center.

\section{Invadopodium assays}

Glass-bottomed dishes were coated with $100 \mu$ l of warm Alexa-Fluor-488conjugated gelatin $(0.2 \mathrm{mg} / \mathrm{ml})$ in PBS containing $2 \%$ sucrose. The coated dishes were dried, fixed with pre-chilled glutaraldehyde solution $(0.5 \%$ in PBS), washed with PBS and then reduced with $5 \mathrm{mg} / \mathrm{ml}$ of sodium borohydride in PBS. The dishes were washed extensively with PBS and then incubated with DMEM containing 10\% FBS and antibiotics for $1 \mathrm{~h}$. Cells were plated at low density onto the dishes and cultured for $24 \mathrm{~h}$, fixed with paraformaldehyde and stained for talin 2 (or talin 1 ) and F-actin. Images were acquired using a total internal reflection fluorescence (TIRF) microscope. To measure the area that an invadopodium covered, the images were converted to 16-bit inverted JPEG format (invadopodia are white spots) using ImageJ. The images were then opened with NIS Elements, thresholded (invadopodium spots were precisely covered by red). A region of interest (ROI) was drawn along the cell edge and the total invadopodium area was measured using NIS Elements.

\section{Acknowledgements}

We thank Dr Berni Wehrle-Haller for his participation in the project discussion; Dr Ken Jacobson (UNC-Chapel Hill) for the technique of traction force assays; Latifeh Azizi (University of Tampere) for her support in affinity assays; Drs Berni Wehrle-Haller, Ken Jacobson and Andrew Morris for critical reading of this manuscript. 
Competing interests

The authors declare no competing or financial interests.

\section{Author contributions}

L.Q., N.J., X.L. and L.L. performed experiments, analyzed data, and wrote the paper; Z.C. instructed the traction force measurement and analysis; V.P.H. and B.T.G. helped with affinity assays and manuscript writing; C.-G.Z. performed computational analysis; C.H. designed experiments, interpreted results and wrote the paper.

\section{Funding}

This work was supported by a start-up fund from Markey Cancer Center; University of Kentucky; and the American Cancer Society Institutional Research Grant [grant number 85-001-22] and Research Scholar Grant [grant number RSG-13-18401CSM] (to C.H.); and a Biotechnology and Biological Sciences Research Council project grant [grant number BB/N007336/1] (to B.T.G.).

\section{Supplementary information}

Supplementary information available online at

http://jcs.biologists.org/lookup/doi/10.1242/jcs.185959.supplemental

\section{References}

Anthis, N. J., Wegener, K. L., Ye, F., Kim, C., Goult, B. T., Lowe, E. D., Vakonakis I., Bate, N., Critchley, D. R., Ginsberg, M. H. et al. (2009). The structure of an integrin/talin complex reveals the basis of inside-out signal transduction. EMBO J. 28, 3623-3632

Atherton, P., Stutchbury, B., Wang, D.-Y., Jethwa, D., Tsang, R., MeilerRodriguez, E., Wang, P., Bate, N., Zent, R., Barsukov, I. L. et al. (2015). Vinculin controls talin engagement with the actomyosin machinery. Nat. Commun. 6 , 10038

Austen, K., Ringer, P., Mehlich, A., Chrostek-Grashoff, A., Kluger, C., Klingner, C., Sabass, B., Zent, R., Rief, M. and Grashoff, C. (2015). Extracellular rigidity sensing by talin isoform-specific mechanical linkages. Nat. Cell Biol. 17 1597-1606.

Bate, N., Gingras, A. R., Bachir, A., Horwitz, R., Ye, F., Patel, B., Goult, B. T. and Critchley, D. R. (2012). Talin contains A C-terminal calpain2 cleavage site important in focal adhesion dynamics. PLoS ONE 7, e34461.

Beaty, B. T. and Condeelis, J. (2014). Digging a little deeper: the stages of invadopodium formation and maturation. Eur. J. Cell Biol. 93, 438-444.

Beaty, B. T., Wang, Y., Bravo-Cordero, J. J., Sharma, V. P., Miskolci, V., Hodgson, L. and Condeelis, J. (2014). Talin regulates moesin-NHE-1 recruitment to invadopodia and promotes mammary tumor metastasis. J. Cell Biol. 205, 737-751.

Bergman, A., Condeelis, J. S. and Gligorijevic, B. (2014). Invadopodia in context Cell Adh. Migr. 8, 273-279.

Butler, J. P., Tolić-Nørrelykke, I. M., Fabry, B. and Fredberg, J. J. (2002). Traction fields, moments, and strain energy that cells exert on their surroundings. Am. J. Physiol. Cell Physiol. 282, C595-C605.

Calderwood, D. A., Zent, R., Grant, R., Rees, D. J. G., Hynes, R. O. and Ginsberg, M. H. (1999). The talin head domain binds to integrin $\beta$ subunit cytoplasmic tails and regulates integrin activation. J. Biol. Chem. 274 28071-28074.

Chen, C., Wang, X., Xiong, X., Liu, Q., Huang, Y., Xu, Q., Hu, J., Ge, G. and Ling, K. (2015). Targeting type I[gamma] phosphatidylinositol phosphate kinase inhibits breast cancer metastasis. Oncogene 34, 4635-4646.

Debrand, E., Conti, F. J., Bate, N., Spence, L., Mazzeo, D., Pritchard, C. A., Monkley, S. J. and Critchley, D. R. (2012). Mice carrying a complete deletion of the talin2 coding sequence are viable and fertile. Biochem. Biophys. Res. Commun. 426, 190-195.

Deng, H., Deng, S., Xu, H., Deng, H.-X., Chen, Y., Yuan, L., Deng, X., Yang, S., Guan, L., Zhang, J. et al. (2016). Exome sequencing of a pedigree reveals S339L mutation in the TLN2 gene as a cause of fifth finger camptodactyly. PLoS ONE 11, e0155180.

Desiniotis, A. and Kyprianou, N. (2011). Chapter four-significance of talin in cancer progression and metastasis. Int. Rev. Cell Mol. Biol. 289, 117-147.

Di Paolo, G., Pellegrini, L., Letinic, K., Cestra, G., Zoncu, R., Voronov, S. Chang, S., Guo, J., Wenk, M. R. and De Camilli, P. (2002). Recruitment and regulation of phosphatidylinositol phosphate kinase type 1[gamma] by the FERM domain of talin. Nature. $\mathbf{4 2 0}, 85-89$

Dumbauld, D. W., Lee, T. T., Singh, A., Scrimgeour, J., Gersbach, C. A., Zamir, E. A., Fu, J., Chen, C. S., Curtis, J. E., Craig, S. W., et al. (2013). How vinculin regulates force transmission. Proc. Natl. Acad. Sci. USA. 110, 9788-9793.

Franco, S. J., Rodgers, M. A., Perrin, B. J., Han, J., Bennin, D. A., Critchley, D. R. and Huttenlocher, A. (2004). Calpain-mediated proteolysis of talin regulates adhesion dynamics. Nat. Cell Biol. 6, 977-983.

Giannone, G., Jiang, G., Sutton, D. H., Critchley, D. R. and Sheetz, M. P. (2003) Talin1 is critical for force-dependent reinforcement of initial integrin-cytoskeleton bonds but not tyrosine kinase activation. J. Cell Biol. 163, 409-419.
Gingras, A. R., Ziegler, W. H., Frank, R., Barsukov, I. L., Roberts, G. C. K., Critchley, D. R. and Emsley, J. (2005). Mapping and consensus sequence identification for multiple vinculin binding sites within the talin rod. J. Biol. Chem. 280, 37217-37224

Goult, B. T., Zacharchenko, T., Bate, N., Tsang, R., Hey, F., Gingras, A. R. Elliott, P. R., Roberts, G. C. K., Ballestrem, C., Critchley, D. R. et al. (2013). RIAM and vinculin binding to talin are mutually exclusive and regulate adhesion assembly and turnover. J. Biol. Chem. 288, 8238-8249.

Hemmings, L., Rees, D. J., Ohanian, V., Bolton, S. J., Gilmore, A. P., Patel, B. Priddle, H., Trevithick, J. E., Hynes, R. O. and Critchley, D. R. (1996). Talin contains three actin-binding sites each of which is adjacent to a vinculin-binding site. J. Cell Sci. 109, 2715-2726.

Hinz, B., and Gabbiani, G. (2003). Mechanisms of force generation and transmission by myofibroblasts. Curr. Opin. Biotechnol. 14, 538-546.

Huang, C., Rajfur, Z., Yousefi, N., Chen, Z., Jacobson, K. and Ginsberg, M. H. (2009). Talin phosphorylation by Cdk5 regulates Smurf1-mediated talin head ubiquitylation and cell migration. Nat. Cell Biol. 11, 624-630.

Jin, J. K., Tien, P. C., Cheng, C. J., Song, J. H., Huang, C., Lin, S.-H. and Gallick G. E. (2015). Talin1 phosphorylation activates [beta]1 integrins: a nove mechanism to promote prostate cancer bone metastasis. Oncogene 34 1811-1821.

Le, X.-F., Almeida, M. I., Mao, W., Spizzo, R., Rossi, S., Nicoloso, M. S., Zhang S., Wu, Y., Calin, G. A. and Bast, R. C.Jr. (2012). Modulation of MicroRNA-194 and cell migration by HER2-targeting trastuzumab in breast cancer. PLOS ONE 7 , e41170

Li, X., Zhou, Q., Sunkara, M., Kutys, M. L., Wu, Z., Rychahou, P., Morris, A. J., Zhu, H., Evers, B. M. and Huang, C. (2013). Ubiquitylation of phosphatidylinositol 4-phosphate 5-kinase type I $\gamma$ by HECTD1 regulates focal adhesion dynamics and cell migration. J. Cell Sci. 126, 2617-2628.

Ling, K., Doughman, R. L., Firestone, A. J., Bunce, M. W. and Anderson, R. A. (2002). Type I[gamma] phosphatidylinositol phosphate kinase targets and regulates focal adhesions. Nature $\mathbf{4 2 0}, 89-93$

Monkley, S. J., Zhou, X.-H., Kinston, S. J., Giblett, S. M., Hemmings, L., Priddle H., Brown, J. E., Pritchard, C. A., Critchley, D. R. and Fässler, R. (2000) Disruption of the talin gene arrests mouse development at the gastrulation stage. Dev. Dyn. 219, 560-574.

Morimatsu, M., Mekhdjian, A. H., Chang, A. C., Tan, S. J., and Dunn, A. R. (2015). Visualizing the Interior Architecture of Focal Adhesions with HighResolution Traction Maps. Nano Lett. 15, 2220-2228.

Nayal, A., Webb, D. J. and Horwitz, A. F. (2004). Talin: an emerging focal point of adhesion dynamics. Curr. Opin. Cell Biol. 16, 94-98.

Nuckolls, G. H., Turner, C. E. and Burridge, K. (1990). Functional-studies of the domains of talin. J. Cell Biol. 110, 1635-1644.

Paz, H., Pathak, N. and Yang, J. (2014). Invading one step at a time: the role of invadopodia in tumor metastasis. Oncogene 33, 4193-4202.

Praekelt, U., Kopp, P. M., Rehm, K., Linder, S., Bate, N., Patel, B., Debrand, E. Manso, A. M., Ross, R. S., Conti, F. et al. (2012). New isoform-specific monoclonal antibodies reveal different sub-cellular localisations for talin1 and talin2. Eur. J. Cell Biol. 91, 180-191.

Ran, F. A., Hsu, P. D., Wright, J., Agarwala, V., Scott, D. A. and Zhang, F. (2013) Genome engineering using the CRISPR-Cas9 system. Nat. Protocols. 8 2281-2308.

Revach, O.-Y. and Geiger, B. (2014). The interplay between the proteolytic invasive, and adhesive domains of invadopodia and their roles in cancer invasion. Cell Adh. Migr. 8, 215-225.

Saykali, B. A. and El-Sibai, M. (2014). Invadopodia, regulation, and assembly in cancer cell invasion. Cell Commun. Adhes. 21, 207-212.

Senetar, M. A., Moncman, C. L. and McCann, R. O. (2007). Talin2 is induced during striated muscle differentiation and is targeted to stable adhesion complexes in mature muscle. Cell Motil. Cytoskeleton. 64, 157-173.

Tadokoro, S., Shattil, S. J., Eto, K., Tai, V., Liddington, R. C., de Pereda, J. M Ginsberg, M. H. and Calderwood, D. A. (2003). Talin binding to integrin ß tails: a final common step in integrin activation. Science 302, 103-106.

Weaver, A. M. (2006). Invadopodia: specialized cell structures for cancer invasion. Clin. Exp. Metastasis 23, 97-105

Webb, D. J., Parsons, J. T. and Horwitz, A. F. (2002). Adhesion assembly, disassembly and turnover in migrating cells-over and over and over again. Nat Cell Biol. 4, E97-E100.

Wehrle-Haller, B. (2012). Assembly and disassembly of cell matrix adhesions. Curr Opin. Cell Biol. 24, 569-581.

Wu, Z., Li, X., Sunkara, M., Spearman, H., Morris, A. J. and Huang, C. (2011) PIPKI $\gamma$ regulates focal adhesion dynamics and colon cancer cell invasion. PLoS ONE 6, e24775.

Yamaguchi, H., Pixley, F. and Condeelis, J. (2006). Invadopodia and podosomes in tumor invasion. Eur. J. Cell Biol. 85, 213-218.

Yu, C.-h., Rafiq, N. B. M., Krishnasamy, A., Hartman, K. L., Jones, G. E., Bershadsky, A. D. and Sheetz, M. P. (2013). Integrin-matrix clusters form podosome-like adhesions in the absence of traction forces. Cell Rep. 5 $1456-1468$ 
Zaidel-Bar, R., Ballestrem, C., Kam, Z. and Geiger, B. (2003). Early molecular events in the assembly of matrix adhesions at the leading edge of migrating cells. J. Cell Sci. 116, 4605-4613.
Zhang, X., Jiang, G., Cai, Y., Monkley, S. J., Critchley, D. R. and Sheetz, M. P. (2008). Talin depletion reveals independence of initial cell spreading from integrin activation and traction. Nat. Cell Biol. 10, 1062-1068. 